\title{
Relative Navigation: A Keyframe-Based Approach for Observable GPS-Degraded Navigation
}

\author{
David O. Wheeler \\ Department of Electrical and Computer Engineering, Brigham Young University, dowheeler@gmail.com \\ Daniel P. Koch \\ Department of Mechanical Engineering, Brigham Young University, danielpkoch@gmail.com \\ James S. Jackson \\ Department of Mechanical Engineering, Brigham Young University, james.s.jackson@byu.edu \\ Timothy W. McLain \\ Department of Mechanical Engineering, Brigham Young University, mclain@byu.edu \\ Randal W. Beard

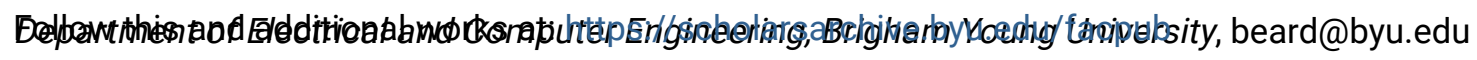 \\ Part of the Electrical and Computer Engineering Commons
}

\section{Original Publication Citation}

D. O. Wheeler, D. P. Koch, J. S. Jackson, T. W. McLain and R. W. Beard, "Relative Navigation: A Keyframe-Based Approach for Observable GPS-Degraded Navigation," in IEEE Control Systems, vol. 38, no. 4, pp. 30-48, Aug. 2018, DOI: 10.1109/MCS.2018.2830079

\section{BYU ScholarsArchive Citation}

Wheeler, David O.; Koch, Daniel P.; Jackson, James S.; McLain, Timothy W.; and Beard, Randal W., "Relative Navigation: A Keyframe-Based Approach for Observable GPS-Degraded Navigation" (2018). Faculty Publications. 1961.

https://scholarsarchive.byu.edu/facpub/1961

This Peer-Reviewed Article is brought to you for free and open access by BYU ScholarsArchive. It has been accepted for inclusion in Faculty Publications by an authorized administrator of BYU ScholarsArchive. For more information, please contact ellen_amatangelo@byu.edu. 


\title{
Relative Navigation A keyframe-based approach for observable GPS-degraded navigation
}

\author{
David O. Wheeler, Daniel P. Koch, James S. Jackson, \\ Timothy W. McLain, Randal W. Beard \\ POC: D. Wheeler (david.wheeler@byu.edu)
}

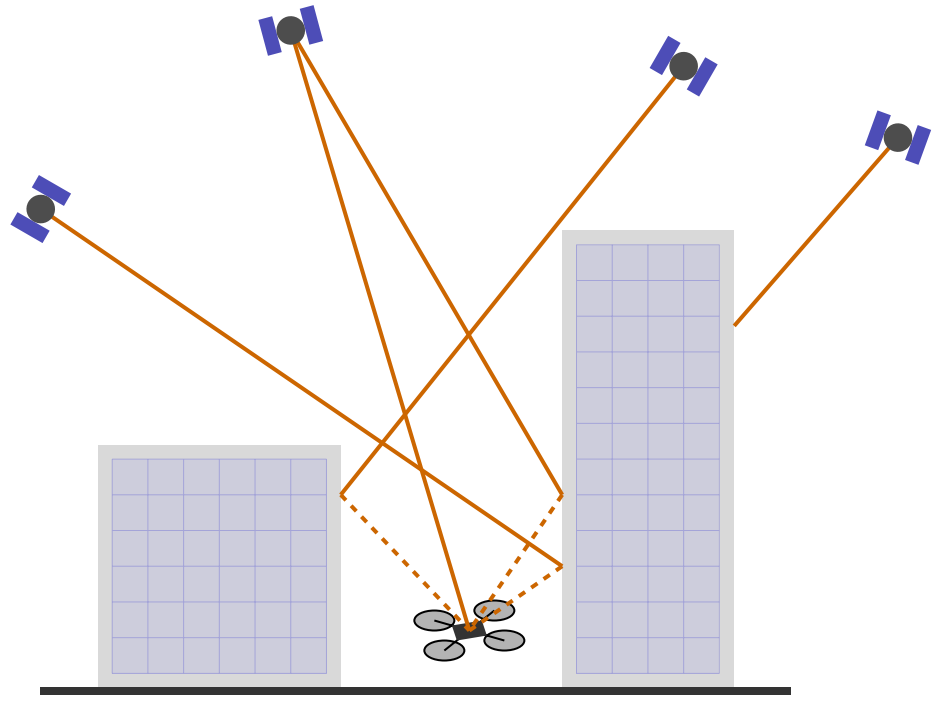

Figure 1: GPS is particularly prone to degradation or dropout when flying near the ground.

As relevant technologies become smaller and less expensive, micro air vehicles (MAVs) are transitioning from predominantly military and hobbyist applications to mainstream use. Exciting new applications include delivery of medical supplies to remote areas, infrastructure inspection, environmental change detection including precision agriculture, surveillance tasks including fire or traffic monitoring, and the film and entertainment industry. However, before MAVs become fully integrated into society and the airspace, higher levels of safety and reliability must be assured.

One of the factors that most limits MAV robustness is their heavy reliance on consistent and accurate measurements from satellite navigation systems such as the Global Positioning System (GPS). These measurements provide regular updates of global position, heading, and velocity, directly influencing state estimation and control. However, GPS is susceptible to degradation and dropout, as illustrated conceptually in Figure 1. The weak signal can be easily blocked by buildings and foliage, jammed, or spoofed. Further, the measurement quality can degrade due to multipath signals, atmospheric delays, or the number and position of visible satellites. These issues are particularly prevalent when flying near the ground, where safety and reliability are especially important.
To circumvent these difficulties, many GPS-denied navigation solutions have been developed that utilize relative measurements from algorithms such as visual odometry or laser scan matching [1-4]. Even with these measurements, however, when GPS is unavailable the global position and heading states are not observable [5,6], as shown in the observability analysis in [7]. This means there is no guarantee that these states can be accurately reconstructed from the available inputs and measurements. Specifically, non-observability induces three main difficulties:

1. Global drift: Integrating noisy inputs without correction will cause the global state to drift arbitrarily far from truth.

2. Estimator inconsistency: An inconsistent estimator is one where either the estimates are biased or the covariance estimate does not well represent the underlying uncertainty distribution.

3. Potential instability: Feedback control typically assumes some level of state observability. There is no guarantee that driving an unobservable estimated state to a desired state will actually stabilize the system.

Current filter-based approaches to GPS-denied navigation directly estimate the global state of the vehicle. In this article we demonstrate for such systems that, because of unobservability, estimation and control performance can degrade significantly during periods of prolonged GPS dropout and heading uncertainty. We present as an alternative the relative navigation framework, which maintains full-state observability in spite of GPS dropout by estimating with respect to a local reference frame. While relative navigation, like all GPS-denied navigation approaches, is subject to global drift, it maintains a more accurate estimate of global uncertainty and so provides better inputs to techniques such as map optimization using loop closures that help to reduce this drift. Relative navigation facilitates consistent state estimation and stable control, thereby improving the overall safety and reliability of MAVs.

This article promotes a paradigm shift within the GPSdenied navigation literature. Many researchers are accustomed to working with respect to a global reference frame, and as a result concede that state observability is inevitably lost in the absence of global updates [5]. This article highlights 
the issues associated with such a concession and provides a viable alternative. In particular, we show that unobservability leads to a loss of estimator consistency. Inconsistency implies a loss of estimator optimality [8]. By subtly restructuring the problem, relative navigation avoids this and other pitfalls that are prevalent in GPS-denied navigation systems. In terms of implementation, the modifications that need to be made to an existing keyframe-based global filter implementation to convert it to relative navigation are relatively minor and straightforward.

We begin the article with a tutorial overview of current state-of-the-art, keyframe-based approaches for GPS-denied MAV navigation. We first summarize the most common global estimation approaches in a side-by-side comparison, highlighting the differences in how they incorporate the relative odometry measurements. We then introduce the relative navigation framework, explaining how it incorporates relative measurements to produce local state estimates, and how it uses these local estimates to produce an estimate of the global pose of the MAV. With these preliminaries established, we then present the main contribution of this article, which is a rigorous analysis and comparison of the performance of each of these estimation approaches in simulation. We show that the relative navigation framework provides advantages in terms of accuracy, consistency, and its ability handle global updates after a prolonged GPS outage. To demonstrate that the findings in the simulation apply to real-world environments, we also present limited multirotor flight-test results for each of the approaches. We then conclude with some final discussion. It should be noted at the outset that the purpose of this article is to compare the performance of different theoretical estimation frameworks, rather than to present the specific details of a new estimator. While a background in Kalman filtering and statistical analysis will be useful to the reader, relevant concepts are reviewed as needed.

\section{State-of-the-Art MAV Navigation}

The majority of autonomous MAVs currently in operation use GPS with an inertial navigation system (GPS/INS) for state estimation and control. High-rate, body-fixed accelerometers and gyroscope measurements are integrated to estimate change in position and attitude. When properly calibrated, these measurements are remarkably accurate over small time steps and are commonly used as inputs to the estimator's dynamics. Integrating noise, however, ultimately causes these estimates to drift. In general, GPS measurements do not drift with time, but are available at a slower rate. Commonly a probabilistic filter, such as an extended Kalman filter (EKF), fuses measurements from these two sensors using a model of the vehicle dynamics. When GPS is available and reliable, GPS/INS solutions work well for global missions requiring position hold or waypoint following. GPS/INS systems have been thoroughly researched and are widely used.

When GPS is unavailable, MAV navigation approaches typically utilize odometry computed from exteroceptive sensors such as cameras or laser scanners. Many such odometry algorithms exist for a variety of sensors, and include methods such as visual odometry $[9,10]$ and laser scan matching $[4,11]$.
Odometry can be computed either between consecutive frames (images or scans), or between the current frame and a keyframe. When a keyframe is used, a series of odometry measurements are computed with respect to this common, fixed reference frame. Typically, the keyframe is updated only when there is insufficient overlap to provide a reliable odometry measurement. As a result, keyframe-based odometry reduces temporal drift in the computed odometry as compared to frame-to-frame matching $[1,3]$. In this paper we focus on global keyframe filters, which build upon GPS/INS solutions but incorporate the relative pose measurements provided by these keyframe-based odometry approaches. Many of the concepts in this paper find parallels in the simultaneous localization and mapping (SLAM) literature. "Connection to Simultaneous Localization and Mapping" briefly summarizes the development of SLAM, including the recent emphasis on improving consistency through relative formulations and efforts to reduce computational complexity.

In the next section we briefly review the extended Kalman filter algorithm in a general sense, and establish the notation that is used throughout this article. We then describe the three global keyframe filters to be analyzed. While the formulation presented in this article is for a discrete-time system, the principles apply to continuous or continuous-discrete formulations as well.

\section{Extended Kalman Filter}

The extended Kalman filter (EKF) is an extension of the Kalman filter to systems with nonlinear dynamics and/or nonlinear measurement models. It recursively estimates the system state $\hat{\mathbf{x}}_{t}$ as a function of the state estimate at the previous time step $\hat{\mathbf{x}}_{t-1}$, inputs $\mathbf{u}_{t}$, and measurements $\mathbf{z}_{t}$. It also maintains an estimate of its uncertainty, represented by the covariance matrix $\mathbf{P}_{t}$.

Consider the discrete-time system

$$
\mathbf{x}_{t}=\mathbf{f}\left(\mathbf{x}_{t-1}, \mathbf{u}_{t}+\boldsymbol{v}_{t}\right)+\boldsymbol{\xi}_{t},
$$

where $\boldsymbol{v}_{t} \sim \mathcal{N}\left(0, \mathbf{Q}_{\mathbf{u}}\right)$ and $\boldsymbol{\xi}_{t} \sim \mathcal{N}\left(0, \mathbf{Q}_{\mathbf{x}}\right)$ are zero-mean Gaussian random variables. In the prediction step, the filter propagates the state estimates forward as

$$
\hat{\mathbf{x}}_{t \mid t-1}=\mathbf{f}\left(\hat{\mathbf{x}}_{t-1 \mid t-1}, \mathbf{u}_{t}\right) .
$$

The covariance is propagated according to the linear approximation

$$
\mathbf{P}_{t \mid t-1}=\mathbf{F}_{t} \mathbf{P}_{t-1 \mid t-1} \mathbf{F}_{t}^{\top}+\mathbf{G}_{t} \mathbf{Q}_{\mathbf{u}} \mathbf{G}_{t}^{\top}+\mathbf{Q}_{\mathbf{x}},
$$

where $\mathbf{F}_{t}$ and $\mathbf{G}_{t}$ are the Jacobians of the system dynamics with respect to the state and input respectively.

The update step is performed after the prediction step when a new measurement $\mathbf{z}$ is available. In practice several prediction steps may be performed between updates. A measurement is modeled as

$$
\mathbf{z}_{t}=\mathbf{h}\left(\mathbf{x}_{t}\right)+\boldsymbol{\zeta}_{t},
$$

where $\boldsymbol{\zeta}_{t} \sim \mathcal{N}(\mathbf{0}, \mathbf{R})$ is zero-mean Gaussian noise. The Kalman gain is computed as

$$
\mathbf{K}_{t}=\mathbf{P}_{t \mid t-1} \mathbf{H}_{t}^{\top}\left(\mathbf{H}_{t} \mathbf{P}_{t \mid t-1} \mathbf{H}_{t}^{\top}+\mathbf{R}\right)^{-1}
$$


Table 1: Estimation framework legend.

\begin{tabular}{cl}
\hline BL & Baseline (propagation only, no vision update) \\
PG & Pseudo-global \\
SC & Stochastic cloning \\
kRC & Keyframe robocentric \\
kRCi & Keyframe robocentric (inertial error) \\
RN & Relative navigation \\
bRN & Relative navigation (body-fixed dynamics) \\
\hline
\end{tabular}

where $\mathbf{H}_{k}$ is the Jacobian of the measurement model with respect to the state. The state and covariance are updated as

$$
\begin{aligned}
\mathbf{x}_{t \mid t} & =\mathbf{x}_{t \mid t-1}+\mathbf{K}_{t}\left(\mathbf{z}_{t}-\mathbf{h}\left(\hat{\mathbf{x}}_{t \mid t-1}\right)\right) \\
\mathbf{P}_{t \mid t} & =\left(\mathbf{I}-\mathbf{K}_{t} \mathbf{H}_{t}\right) \mathbf{P}_{t \mid t-1}\left(\mathbf{I}-\mathbf{K}_{t} \mathbf{H}_{t}\right)^{\top}+\mathbf{K}_{t} \mathbf{R} \mathbf{K}_{t}^{\top},
\end{aligned}
$$

using Joseph's form to avoid numerical instability [8].

Several of the estimation approaches examined in this article also utilize a third step that we refer to as marginalization. When the odometry algorithm declares a new keyframe, portions of the state vector are reset. We express this operation as

$$
\hat{\mathbf{x}}^{+}=\mathbf{m}(\hat{\mathbf{x}}) .
$$

The covariance is also updated according to the linear approximation

$$
\mathbf{P}^{+}=\mathbf{M P M}^{\top}
$$

where $\mathbf{M}=\partial \mathbf{m} / \partial \mathbf{x}$ is the Jacobian of the marginalization operation with respect to the state.

\section{Global Estimation Approaches}

The three types of global keyframe filters that we examine in this article are pseudo-global, stochastic cloning, and robocentric. These methods all utilize the EKF paradigm, but differ in the way they use the relative odometry measurements to update the global state estimate. In the following sections we describe the internal states, propagation and measurement models, and marginalization steps used by each filter. This information is also presented graphically in Figure 2. The acronyms used throughout the article to refer to each approach are summarized in Table 1.

To simplify the presentation, it is assumed that the vehicle's dynamics with respect to some arbitrary, inertial reference frame can be described by the function $\mathbf{f}_{\mathbf{x}}$, which is a specific instantiation of (1). The Jacobians of these dynamics with respect to the state and input noise is notated as $\mathbf{F}_{\mathbf{x}}$ and $\mathbf{G}_{\mathbf{x}}$ respectively.

An operation that we use with some frequency is compounding the poses represented by two states. Using notation similar to [12], we denote this operation using the $\oplus$ operator as

$$
\mathbf{x}=\mathbf{x}^{a} \oplus \mathbf{x}^{b} .
$$

for frames $a$ and $b$. We also commonly wish to take the Jacobian of this operation for two poses $\hat{\mathbf{x}}^{a}$ and $\hat{\mathbf{x}}^{b}$. For compact- ness we define the Jacobians

$$
\begin{aligned}
& \left.\mathbf{J}_{1 \oplus}\right|_{\hat{\mathbf{x}}^{a}, \hat{\mathbf{x}}^{b}}=\left.\frac{\partial\left(\mathbf{x}^{a} \oplus \mathbf{x}^{b}\right)}{\partial \mathbf{x}^{a}}\right|_{\hat{\mathbf{x}}^{a}, \hat{\mathbf{x}}^{b}}, \\
& \left.\mathbf{J}_{2 \oplus}\right|_{\hat{\mathbf{x}}^{a}, \hat{\mathbf{x}}^{b}}=\left.\frac{\partial\left(\mathbf{x}^{a} \oplus \mathbf{x}^{b}\right)}{\partial \mathbf{x}^{b}}\right|_{\hat{\mathbf{x}}^{a}, \hat{\mathbf{x}}^{b}} .
\end{aligned}
$$

We also commonly reverse the direction of the first pose in the compounding operation. This reversal is denoted by the $\ominus$ operator, so that in the expression

$$
\mathbf{x}=\ominus \mathbf{x}^{a} \oplus \mathbf{x}^{b},
$$

the reversal of $\mathbf{x}^{a}$ is compounded with $\mathbf{x}^{b}$. The Jacobian of the reversal operation for some pose $\hat{\mathbf{x}}$ is defined as

$$
\left.\mathbf{J}_{\ominus}\right|_{\hat{\mathbf{x}}}=\left.\frac{\partial(\ominus \mathbf{x})}{\partial \mathbf{x}}\right|_{\hat{\mathbf{x}}} .
$$

With these definitions, it is then the case that

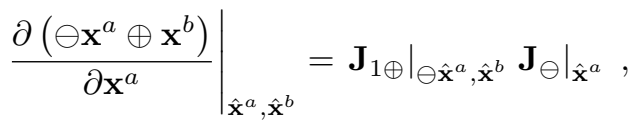

and

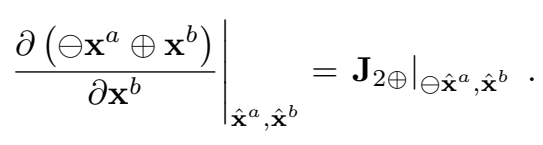

As an example, for the 2D planar case where a pose is represented by the three-vector $\mathbf{x}^{i}=\left[\begin{array}{lll}x_{i} & y_{i} & \phi_{i}\end{array}\right]^{\top}$, these operations and Jacobians are

$$
\begin{aligned}
& \mathbf{x}^{a} \oplus \mathbf{x}^{b}=\left[\begin{array}{c}
x_{a}+x_{b} \cos \phi_{a}-y_{b} \sin \phi_{a} \\
y_{a}+x_{b} \sin \phi_{a}+y_{b} \cos \phi_{a} \\
\phi_{a}+\phi_{b}
\end{array}\right], \\
& \ominus \mathbf{x}^{a}=\left[\begin{array}{c}
-x_{a} \cos \phi_{a}-y_{a} \sin \phi_{a} \\
x_{a} \sin \phi_{a}-y_{a} \cos \phi_{a} \\
-\phi_{a}
\end{array}\right] \text {, } \\
& \mathbf{J}_{1 \oplus \mathbf{x}^{a}, \mathbf{x}^{b}}=\left[\begin{array}{ccc}
1 & 0 & -x_{b} \sin \phi_{a}-y_{b} \cos \phi_{a} \\
0 & 1 & x_{b} \cos \phi_{a}-y_{b} \sin \phi_{a} \\
0 & 0 & 1
\end{array}\right] \text {, } \\
& \mathbf{J}_{\left.2 \oplus\right|_{\mathbf{x}^{a}, \mathbf{x}^{b}}}=\left[\begin{array}{ccc}
\cos \phi_{a} & -\sin \phi_{a} & 0 \\
\sin \phi_{a} & \cos \phi_{a} & 0 \\
0 & 0 & 1
\end{array}\right], \\
& \left.\mathbf{J}_{\ominus}\right|_{\mathbf{x}^{a}}=\left[\begin{array}{ccc}
-\cos \phi_{a} & -\sin \phi_{a} & x_{a} \sin \phi_{a}-y_{a} \cos \phi_{a} \\
\sin \phi_{a} & -\cos \phi_{a} & x_{a} \cos \phi_{a}+y_{a} \sin \phi_{a} \\
0 & 0 & -1
\end{array}\right] \text {. }
\end{aligned}
$$

\section{Pseudo-Global}

The pseudo-global (PG) approach, illustrated in Figure 2a, is perhaps the simplest and most intuitive of the methods, but is technically incorrect and has significant theoretical shortcomings. While it produces surprisingly accurate estimates, it becomes grossly overconfident because it ignores important cross-correlation terms. We include it here because several prominent research groups have used this approach for MAV navigation $[4,13]$. 


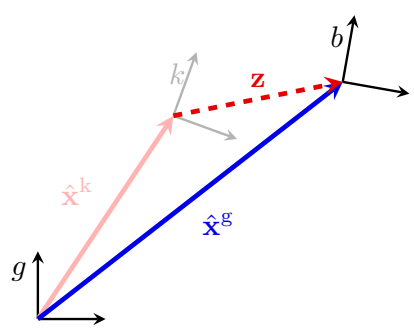

(a) Pseudo-global

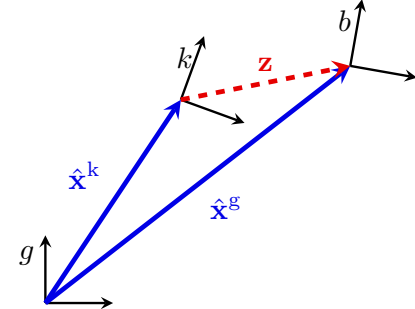

(b) Stochastic cloning

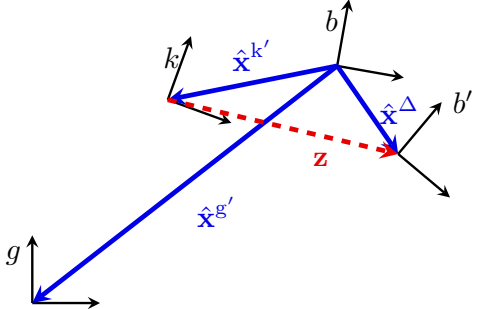

(c) Keyframe robocentric

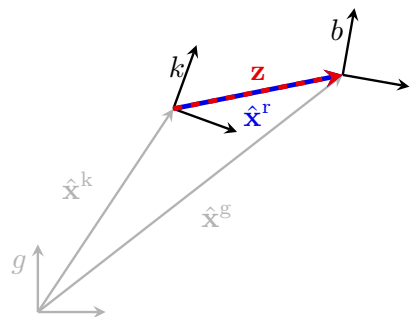

(d) Relative navigation

Figure 2: Comparison of estimator architectures. Pseudo-global (PG) compounds the keyframe state and measurement to apply a global-like update. Stochastic cloning (SC) estimates both the global state and keyframe state and applies the update as the relative difference. Keyframe robocentric $(\mathrm{kRC})$ reverses the state direction and uses a delta state for applying the prediction and update steps. Relative navigation (RN) only estimates the relative state. The coordinate frames labeled $g, k$, and $b$ correspond to the global, keyframe, and body frame of the robot.

In one embodiment of this approach, the vision sensor is fed into a monocular-SLAM framework, whose output is treated as a black-box global pose update that is fused directly in the filter $[13,14]$. In other implementations $[4,15]$, the relative measurement is applied by appending it to a saved estimate of the keyframe's global position $\hat{\mathbf{x}}$ and then treating it as a global measurement. In this case the estimated state is simply the global state of the vehicle,

$$
\hat{\mathbf{x}}_{\mathrm{PG}}=\hat{\mathbf{x}}^{\mathrm{g}}, \quad \mathbf{P}_{\mathrm{PG}}=\mathbf{P}^{\mathrm{g}},
$$

and the propagation equations are the vehicle dynamics

$$
\begin{gathered}
\mathbf{f}_{\mathrm{PG}}\left(\hat{\mathbf{x}}_{\mathrm{PG}}, \mathbf{u}\right)=\mathbf{f}_{\mathbf{x}}\left(\hat{\mathbf{x}}^{\mathrm{g}}, \mathbf{u}\right), \\
\mathbf{F}_{\mathrm{PG}}=\left.\mathbf{F}_{\mathbf{x}}\right|_{\hat{\mathbf{x}}^{\mathrm{g}}}, \quad \mathbf{G}_{\mathrm{PG}}=\left.\mathbf{G}_{\mathbf{x}}\right|_{\hat{\mathbf{x}}^{\mathrm{g}}} .
\end{gathered}
$$

The pseudo-global measurement is obtained by compounding the saved estimate of the keyframe state with the relative measurement obtained from the odometry as

$$
\mathbf{z}_{\mathrm{PG}}=\hat{\mathbf{x}}^{\mathrm{k}} \oplus \mathbf{z} .
$$

An estimate of the measurement covariance is also needed. The most naive approach is to simply use the covariance for the relative odometry measurement. However, this method ignores uncertainty in the saved keyframe state $\hat{\mathbf{x}}^{\mathrm{k}}$ used to construct the measurement. A slightly better approach is to use the covariance of (7), which is

$$
\mathbf{R}_{\mathrm{PG}}=\left(\left.\mathbf{J}_{1 \oplus}\right|_{\hat{\mathbf{x}}^{\mathrm{k}}, \mathbf{z}}\right) \mathbf{P}^{\mathrm{k}}\left(\left.\mathbf{J}_{1 \oplus}\right|_{\hat{\mathbf{x}}^{\mathrm{k}}, \mathbf{z}}\right)^{\top}+\left(\left.\mathbf{J}_{2 \oplus}\right|_{\hat{\mathbf{x}}^{\mathrm{k}}, \mathbf{z}}\right) \mathbf{R}\left(\left.\mathbf{J}_{2 \oplus}\right|_{\hat{\mathbf{x}}^{\mathrm{k}}, \mathbf{z}}\right)^{\top} .
$$

When the measurement is constructed in this fashion, the predicted measurement is then simply the global state of the vehicle

$$
\begin{aligned}
\mathbf{h}_{\mathrm{PG}}\left(\hat{\mathbf{x}}_{\mathrm{PG}}\right) & =\hat{\mathbf{x}}^{\mathrm{g}}, \\
\mathbf{H}_{\mathrm{PG}} & =\mathbf{I} .
\end{aligned}
$$

While the modified measurement covariance described above incorporates uncertainty in the saved estimate of the keyframe state, it ignores cross-correlation terms between the keyframe state and the current global pose. In addition, treating a relative measurement as a direct update to the global state in this fashion causes the estimator to become grossly overconfident, as will be demonstrated in the results section of this article.

\section{Stochastic Cloning}

The stochastic cloning (SC) approach [16] improves on the pseudo-global approach by accounting for the uncertainty in the global pose of the keyframe as well as the crosscorrelations with the vehicle's global pose. In this approach, illustrated by Figure 2b, the state and covariance are augmented with the global pose of the keyframe $\mathbf{x}^{\mathrm{k}}$ as

$$
\hat{\mathbf{x}}_{\mathrm{SC}}=\left[\begin{array}{c}
\hat{\mathbf{x}}^{\mathrm{g}} \\
\hat{\mathbf{x}}^{\mathrm{k}}
\end{array}\right], \quad \mathbf{P}_{\mathrm{SC}}=\left[\begin{array}{cc}
\mathbf{P}^{\mathrm{g}} & \mathbf{P}^{\mathrm{g}, \mathrm{k}} \\
\mathbf{P}^{\mathrm{k}, \mathrm{g}} & \mathbf{P}^{\mathrm{k}}
\end{array}\right],
$$

where $\mathbf{P}^{\mathrm{g}}$ is the uncertainty in the global state, $\mathbf{P}^{\mathrm{k}}$ is the uncertainty in the keyframe state, and $\mathbf{P}^{\mathrm{g}, \mathrm{k}}$ is the crosscorrelation. During the prediction step, the keyframe state is not expected to change since there is no new information about it. The propagation equations therefore become

$$
\begin{gathered}
\mathbf{f}_{\mathrm{SC}}\left(\hat{\mathbf{x}}_{\mathrm{SC}}, \mathbf{u}\right)=\left[\begin{array}{c}
\mathbf{f}_{\mathbf{x}}\left(\hat{\mathbf{x}}^{\mathrm{g}}, \mathbf{u}\right) \\
\hat{\mathbf{x}}^{\mathrm{k}}
\end{array}\right], \\
\mathbf{F}_{\mathrm{SC}}=\left[\begin{array}{cc}
\left.\mathbf{F}_{\mathbf{x}}\right|_{\hat{\mathbf{x}}^{\mathrm{g}}} & \mathbf{0} \\
\mathbf{0} & \mathbf{I}
\end{array}\right], \quad \mathbf{G}_{\mathrm{SC}}=\left[\begin{array}{c}
\left.\mathbf{G}_{\mathbf{x}}\right|_{\hat{\mathbf{x}}^{\mathrm{g}}} \\
\mathbf{0}
\end{array}\right] .
\end{gathered}
$$

The predicted measurement is obtained by compounding the inverse keyframe state with the global vehicle state to estimate the relative measurement from the keyframe to the body. The measurement model is then

$$
\mathbf{h}_{\mathrm{SC}}\left(\hat{\mathbf{x}}_{\mathrm{SC}}\right)=\ominus \hat{\mathbf{x}}^{\mathrm{k}} \oplus \hat{\mathbf{x}}^{\mathrm{g}},
$$

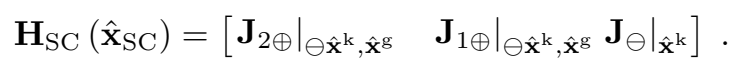

Each time the odometry algorithm declares a new keyframe, the estimator's keyframe state must be updated. The old keyframe state is marginalized out, and the new keyframe state is initialized as the current estimate of the vehicle's global state:

$$
\begin{gathered}
\mathbf{m}_{\mathrm{SC}}\left(\hat{\mathbf{x}}_{\mathrm{SC}}\right)=\left[\begin{array}{c}
\hat{\mathbf{x}}^{\mathrm{g}} \\
\hat{\mathbf{x}}^{\mathrm{g}}
\end{array}\right], \\
\mathbf{M}_{\mathrm{SC}}\left(\hat{\mathbf{x}}_{\mathrm{SC}}\right)=\left[\begin{array}{ll}
\mathbf{I} & 0 \\
\mathbf{I} & 0
\end{array}\right] .
\end{gathered}
$$

The stochastic cloning approach is much more theoretically sound than PG. However, as will be demonstrated in the results section of this article, it still suffers from estimator inconsistency. One reason for this is that the linearization about 
unobservable, drifted global states in the measurement update artificially introduces extra information to the filter [17]. While some methods for addressing this shortcoming have been proposed [18], we demonstrate that the fundamental, underlying issue of unobservability can be avoided entirely with relative navigation approach.

\section{Keyframe-Robocentric}

Robocentric mapping [19] is a more recent approach that addresses many of the consistency issues observed in EKFSLAM approaches that express the vehicle and feature locations with respect to a fixed frame. Robocentric mapping improves consistency by expressing feature locations with respect to a body-fixed - or robocentric - frame, thus reducing the linearization errors that lead to inconsistency.

To the authors' knowledge, robocentric mapping has to date been applied exclusively to the EKF-SLAM problem, where the locations of many features are tracked as states in the filter. To facilitate comparison with the other approaches, we adapt it to a keyframe-based approach by treating the keyframe as the only feature being tracked in the filter. We refer to this adaptation as keyframe-robocentric ( $\mathrm{kRC}$ ) to differentiate from the existing literature.

The state vector for the $\mathrm{kRC}$ filter, illustrated in Figure 2c, consists of the location of the global origin expressed in the body-fixed frame $\hat{\mathbf{x}}^{\mathrm{g}^{\prime}}$, the location of the keyframe expressed in the body-fixed frame $\hat{\mathbf{x}}^{\mathrm{k}^{\prime}}$, and the displacement state $\hat{\mathbf{x}}^{\Delta}$ :

$$
\hat{\mathbf{x}}_{\mathrm{kRC}}=\left[\begin{array}{c}
\hat{\mathbf{x}}^{\mathrm{g}^{\prime}} \\
\hat{\mathbf{x}}^{\mathrm{k}^{\prime}} \\
\hat{\mathbf{x}}^{\Delta}
\end{array}\right], \quad \mathbf{P}_{\mathrm{kRC}}=\left[\begin{array}{ccc}
\mathbf{P}^{\mathrm{g}^{\prime}} & \mathbf{P}^{\mathrm{g}^{\prime}, \mathrm{k}^{\prime}} & \mathbf{P}^{\mathrm{g}^{\prime}, \Delta} \\
\mathbf{P}^{\mathrm{k}^{\prime}, \mathrm{g}^{\prime}} & \mathbf{P}^{\mathrm{k}^{\prime}} & \mathbf{P}^{\mathrm{k}^{\prime}, \Delta} \\
\mathbf{P}^{\Delta, \mathrm{g}^{\prime}} & \mathbf{P}^{\Delta, \mathrm{k}^{\prime}} & \mathbf{P}^{\Delta}
\end{array}\right]
$$

The displacement state represents how the vehicle has moved since the last relative measurement. It is computed by aggregating the changes produced by the prediction equations. Only $\hat{\mathbf{x}}^{\Delta}$ is updated during the prediction step, so that

$$
\begin{gathered}
\mathbf{f}_{\mathrm{kRC}}\left(\hat{\mathbf{x}}_{\mathrm{kRC}}, \mathbf{u}\right)=\left[\begin{array}{c}
\hat{\mathbf{x}}^{\mathrm{g}^{\prime}} \\
\hat{\mathbf{x}}^{\mathrm{k}^{\prime}} \\
\mathbf{f}_{\mathbf{x}}\left(\hat{\mathbf{x}}^{\Delta}, \mathbf{u}\right)
\end{array}\right], \\
\mathbf{F}_{\mathrm{kRC}}=\left[\begin{array}{ccc}
\mathbf{I} & \mathbf{0} & \mathbf{0} \\
\mathbf{0} & \mathbf{I} & \mathbf{0} \\
\mathbf{0} & \mathbf{0} & \left.\mathbf{F}_{\mathbf{x}}\right|_{\hat{\mathbf{x}}^{\Delta}}
\end{array}\right], \quad \mathbf{G}_{\mathrm{kRC}}=\left[\begin{array}{c}
\mathbf{0} \\
\mathbf{0} \\
\left.\mathbf{G}_{\mathbf{x}}\right|_{\hat{\mathbf{x}}^{\Delta}}
\end{array}\right] .
\end{gathered}
$$

The measurement model consists of the inverse of the keyframe state composed with the current displacement state:

$$
\begin{aligned}
& \mathbf{h}_{\mathrm{kRC}}\left(\hat{\mathbf{x}}_{\mathrm{kRC}}\right)=\ominus \hat{\mathbf{x}}^{\mathrm{k}^{\prime}} \oplus \hat{\mathbf{x}}^{\Delta},
\end{aligned}
$$

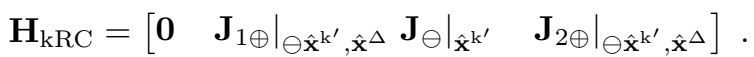

After the update has been applied, the displacement state is compounded onto the global and keyframe states, then reset to zero. This is accomplished with a state marginalization of the form

$$
\mathbf{m}_{\mathrm{kRC}, \mathbf{z}}\left(\hat{\mathbf{x}}_{\mathrm{kRC}}\right)=\left[\begin{array}{c}
\ominus \hat{\mathbf{x}}^{\Delta} \oplus \hat{\mathbf{x}}^{\mathrm{g}^{\prime}} \\
\ominus \hat{\mathbf{x}}^{\Delta} \oplus \hat{\mathbf{x}}^{\mathrm{k}^{\prime}} \\
\mathbf{0}
\end{array}\right]
$$

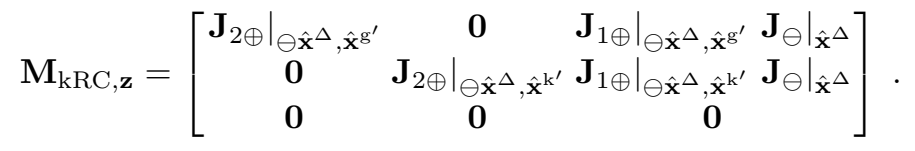

The purpose of this delayed composition is to ensure that the Jacobians associated with the composition, $\mathbf{M}_{\mathrm{kRC}, \mathbf{z}}$, are evaluated only after correction by a measurement update to reduce linearization errors.

Another state augmentation and marginalization also must be performed when a new keyframe is declared. Because the location of the keyframe is expressed in the body frame, and because the body frame was exactly at the location of the keyframe at the time it was declared, this procedure consists of setting the keyframe state to zero with no uncertainty:

$$
\begin{gathered}
\mathbf{m}_{\mathrm{kRC}, \mathrm{k}}\left(\hat{\mathbf{x}}_{\mathrm{kRC}}\right)=\left[\begin{array}{c}
\hat{\mathbf{x}}^{\mathrm{g}^{\prime}} \\
\mathbf{0} \\
\hat{\mathbf{x}}^{\Delta}
\end{array}\right], \\
\mathbf{M}_{\mathrm{kRC}, \mathrm{k}}=\left[\begin{array}{lll}
\mathbf{I} & \mathbf{0} & \mathbf{0} \\
\mathbf{0} & \mathbf{0} & \mathbf{0} \\
\mathbf{0} & \mathbf{0} & \mathbf{I}
\end{array}\right] .
\end{gathered}
$$

As a post-processing operation, it is also common to transform the kRC global state into an inertial frame, denoted $\mathrm{kRCi}$, for purposes such as plotting and path planning. This is accomplished as

$$
\begin{aligned}
\hat{\mathbf{x}}_{\mathrm{kRCi}}^{g} & =\ominus \hat{\mathbf{x}}_{\mathrm{kRC}}^{\mathrm{g}^{\prime}}, \\
\mathbf{P}_{\mathrm{kRCi}} & =\left.\left.\mathbf{J}_{\ominus}\right|_{\hat{\mathbf{x}}} \mathbf{P}_{\mathrm{kRC}} \mathbf{J}_{\ominus}^{\top}\right|_{\hat{\mathbf{x}}} .
\end{aligned}
$$

Note that $\mathbf{P}_{\mathrm{kRCi}}$ is simply a rotation of $\mathbf{P}_{\mathrm{kRC}}$.

\section{Relative Navigation}

The relative navigation $(\mathrm{RN})$ approach addresses the unobservability of the global state by not estimating it in the filter. Instead, only the relative state of the vehicle with respect to the last keyframe is estimated, as illustrated in Figure 2d. The result is that the odometry provides a direct measurement of the state, making the state observable by construction. The global state can be subsequently reconstructed in a back-end map by composing together the series of relative pose estimates produced by the filter. This effectively removes the uncertainty in the global pose from the filter and hands it off to the back end, and as a result the uncertainty of the relative state in the filter remains bounded and consistent. In addition, the pose-graph representation of the global state in the back end creates a more accurate representation of the global uncertainty than can be obtained when representing the global state directly (see "The Banana Distribution").

Another key advantage of the $\mathrm{RN}$ approach is the loose coupling between the relative front end and the global back end, illustrated by the architecture shown in Figure 3. The intuition for this can be developed by imagining a human driver navigating through a city with respect to their local environment - traffic lanes and other vehicles - while opportunistically incorporating local guidance such as "take the 
Under Review. Submitted to IEEE Control System Magazine on Jan 19, 2017.

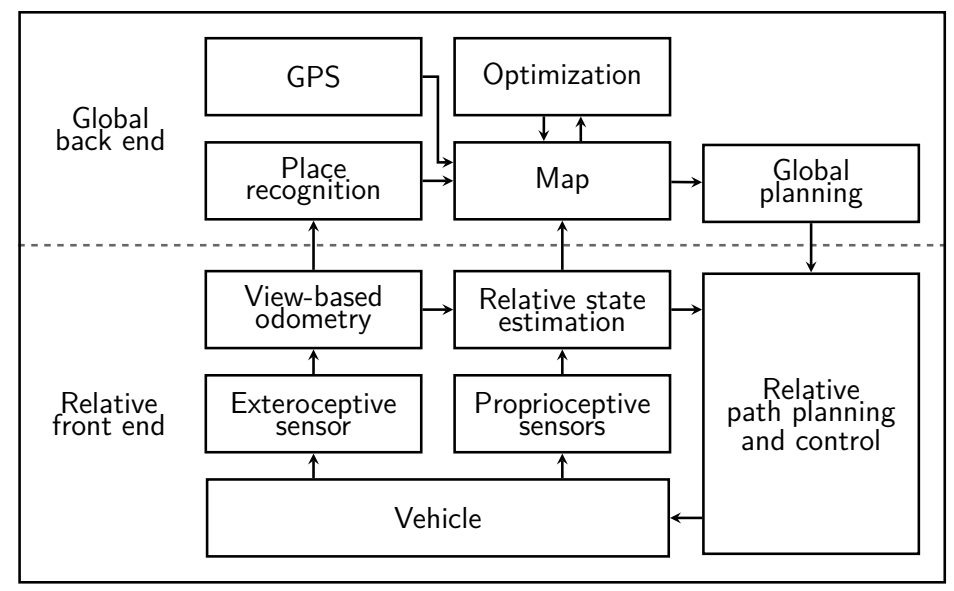

Figure 3: The vehicle-mounted sensors feed a probabilistic filter estimating the vehicle's state relative to its local environment. Here "view-based odometry" refers to algorithms such as visual odometry and laser scan matching. Local path planning and control stabilize the vehicle in this local frame. Meanwhile, on a distinct thread, a time history of local information can be fused with any available global information to form a global map. The back end only influences the flightcritical front end in the form of global goals represented in the current relative coordinate frame.

next right turn" from a navigator in the passenger seat. Similarly, with the relative navigation approach, a vehicle is able to maintain stable flight and avoid collisions indefinitely, even when no global information is available, by using the frontend relative state estimates for local guidance and control. When back-end estimates of the global state change dramatically due to new information, this merely results in a new relative goal being passed to the front end, avoiding the large spikes in control effort seen by systems that control using the global state directly. The system is similarly insulated from delayed or degraded global information.

It is important to note that the general framework in Figure 3 is agnostic to a particular platform, sensor suite, or class of estimation filter. As such, the RN approach can be readily applied to existing systems. The following sections give highlevel descriptions of the relative front end and global back end. For further details on the RN approach and its implementation, the reader is referred to [20-22].

\section{Relative Front End}

The central component of the front end is a filter that estimates the local state of the vehicle. The filter state is the relative pose of the vehicle with respect to the last keyframe, as illustrated in Figure 2d:

$$
\hat{\mathbf{x}}_{\mathrm{RN}}=\hat{\mathbf{x}}^{\mathrm{r}}, \quad \mathbf{P}_{\mathrm{RN}}=\mathbf{P}^{\mathrm{r}} .
$$

During the prediction step the relative state evolves according to the vehicle dynamics

$$
\begin{gathered}
\mathbf{f}_{\mathrm{RN}}\left(\hat{\mathbf{x}}_{\mathrm{RN}}, \mathbf{u}\right)=\mathbf{f}_{\mathbf{x}}\left(\hat{\mathbf{x}}^{\mathrm{r}}, \mathbf{u}\right), \\
\mathbf{F}_{\mathrm{RN}}=\left.\mathbf{F}_{\mathbf{x}}\right|_{\hat{\mathbf{x}}^{\mathrm{r}}}, \quad \mathbf{G}_{\mathrm{RN}}=\left.\mathbf{G}_{\mathbf{x}}\right|_{\hat{\mathbf{x}}^{\mathrm{r}}} .
\end{gathered}
$$

The measurement model is simply the current relative state,

$$
\mathbf{h}_{\mathrm{RN}}\left(\hat{\mathbf{x}}_{\mathrm{RN}}\right)=\hat{\mathbf{x}}^{\mathrm{r}} .
$$

The Jacobian of this measurement model is constant, eliminating linearization errors during the update step:

$$
\mathbf{H}_{\mathrm{RN}}=\mathbf{I} \text {. }
$$

When a new keyframe is declared, the filter first passes its current relative pose estimate and estimated covariance to the back end, then resets its relative state. Because we know that the vehicle was by definition at the location at which the keyframe is declared, the relative state can be reset identically to zero with zero uncertainty as

$$
\begin{gathered}
\mathbf{m}_{\mathrm{RN}}\left(\mathbf{x}_{\mathrm{RN}}\right)=\mathbf{0}, \\
\mathbf{M}_{\mathrm{RN}}=\mathbf{0} .
\end{gathered}
$$

Therefore at each new keyframe, the filter starts with zero pose uncertainty, helping to maintain filter consistency [23]. This concept parallels ideas found in the relative SLAM literature $[24,25]$.

In addition to the estimator, the front end is also responsible for the real-time control of the vehicle. All path planning and control are carried out in the current local frame of the estimator, using goals represented in that coordinate system that come from the back-end global planner. This separation of path planning and control from the global states insulates the system from the large shifts in global state that can occur when loop closure or GPS measurements are obtained and avoids spikes in control effort.

\section{Global Back End}

Each time the front-end estimator resets its state at a new keyframe, it first passes its current estimate of that state and the associated covariance to the back end. These relative pose estimates and covariances from the front-end filter comprise the edges of a pose-graph map. By compounding these edges, the global pose of the vehicle can be computed at any time [26].

Each edge in the graph has a Gaussian uncertainty associated with it, parameterized by the covariance matrix. Compounding these Gaussian uncertainties results in a bananashaped distribution for the uncertainty in the vehicle's global pose. The sidebar "The Banana Distribution" explains that representing the vehicle's global pose as a series of small transforms with Gaussian uncertainty results in a better representation of the global uncertainty than can be obtained by representing the global pose directly.

The pose graph map can also be used to incorporate additional information as it becomes available, such as loop closures or intermittent GPS measurements. These measurements are represented as additional edges in the pose graph, which over-constrains the graph. Optimization methods then adjust the edges in the graph to reduce drift [27,28]. Global mission planning is carried out using this optimized map.

For the purposes of this paper, these additional sources of global information will not be incorporated to facilitate comparison with the other estimation approaches. The global 
results for relative navigation presented in this paper are produced using a limited back end that simply compounds the relative poses and uncertainties provided by the front-end estimator.

\section{Simulation}

A comparison of each of the estimation approaches (PG, SC, $\mathrm{kRC}$, and $\mathrm{RN}$ ) was performed in simulation. Since each of these approaches has previously been demonstrated to work on real MAV platforms, the purpose of the simulation is to compare the theoretical performance of the approaches rather than to evaluate in isolation the suitability of any one approach for MAV navigation. To accomplish this comparison, a simple simulation environment was chosen so as not to obscure the underlying trends. Specifically, simple dynamics and measurement models were used, and process and measurement noise were drawn from known normal distributions. The authors postulate that if an approach breaks down theoretically in an idealized simulation environment, it will perform no better under the complications that arise on an actual MAV platform. The extension to MAV hardware is explored later in the article.

The simulated vehicle is an idealized ground robot following the standard unicycle model. The state of the vehicle is position and heading,

$$
\mathbf{x}=\left[\begin{array}{l}
x \\
y \\
\phi
\end{array}\right]
$$

A random reference trajectory of duration $t_{f}$ is defined by generating forward velocities and angular rates at a rate of $f_{\mathbf{u}}$ and applying them to the dynamics

$$
\dot{\mathbf{x}}_{t}=\left[\begin{array}{c}
\dot{x} \\
\dot{y} \\
\dot{\phi}
\end{array}\right]=\left[\begin{array}{c}
V_{t}^{r} \cos \phi \\
V_{t}^{r} \sin \phi \\
\omega_{t}^{r}
\end{array}\right],
$$

where

$$
\begin{aligned}
V_{t}^{r} & \sim \mathcal{N}\left(V_{\text {nominal }}, \eta_{V}^{2}\right), \\
\omega_{t}^{r} & \sim \mathcal{N}\left(\omega_{t-1}^{r}, \eta_{\omega}^{2}\right) .
\end{aligned}
$$

Identical noisy inputs $\mathbf{u}$ are provided to each estimator to simulate wheel odometry, where

$$
\mathbf{u} \triangleq\left[\begin{array}{l}
V \\
\omega
\end{array}\right]=\left[\begin{array}{c}
V^{r} \\
\omega^{r}
\end{array}\right]+\boldsymbol{v}, \quad \boldsymbol{v} \sim \mathcal{N}\left(\mathbf{0}, \mathbf{Q}_{\mathbf{u}}\right),
$$

with $\mathbf{Q}_{\mathbf{u}}=\operatorname{diag}\left(\sigma_{V}^{2}, \sigma_{\omega}^{2}\right)$. The estimated vehicle dynamics over a time step $\Delta t$ are defined as

$$
\mathbf{f}_{\mathbf{x}}(\hat{\mathbf{x}}, \mathbf{u})=\left[\begin{array}{c}
\hat{x}+V \Delta t \cos \hat{\phi} \\
\hat{y}+V \Delta t \sin \hat{\phi} \\
\hat{\phi}+\omega \Delta t
\end{array}\right]
$$

with Jacobians

$$
\mathbf{F}_{\mathbf{x}}=\left[\begin{array}{ccc}
1 & 0 & -V \Delta t \sin \hat{\phi} \\
0 & 1 & V \Delta t \cos \hat{\phi} \\
0 & 0 & 0
\end{array}\right], \quad \mathbf{G}_{\mathbf{x}}=\left[\begin{array}{cc}
\Delta t \cos \hat{\phi} & 0 \\
\Delta t \sin \hat{\phi} & 0 \\
0 & \Delta t
\end{array}\right]
$$

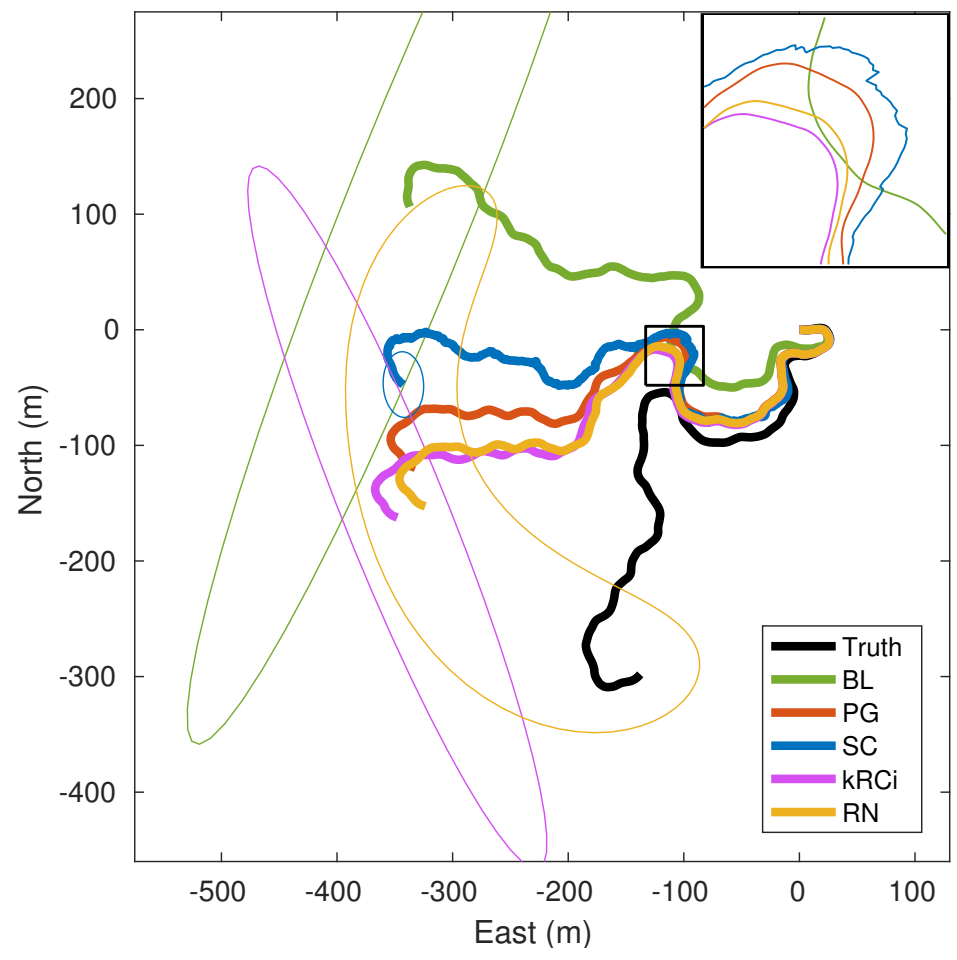

Figure 4: Example simulation for a single trial showing the trajectory estimated by each approach. 90 percent confidence bounds are drawn for each using the global uncertainty covariance. Only the banana-like distribution available with RN contained the true position. The uncertainty bounds for PG are too small to see. The inset image shows the jaggedness of the SC estimates.

Only input noise is added to the dynamic propagation in the simulation, that is $\mathbf{Q}_{\mathbf{x}}=\mathbf{0}$.

Keyframes are established at a rate of $f_{k}$ as the vehicle moves through the environment. Relative measurements are then simulated at a rate of $f_{\mathbf{z}}$ by differencing the keyframe's true pose from the vehicle's current true pose, and then applying Gaussian white noise with covariance $\mathbf{R}=$ $\operatorname{diag}\left(\sigma_{x}^{2}, \sigma_{y}^{2}, \sigma_{\phi}^{2}\right)$.

The estimators use the true values of $\mathbf{Q}_{\mathbf{u}}, \mathbf{Q}_{\mathbf{x}}$, and $\mathbf{R}$, allowing them to produce the best estimates they are theoretically capable of for the given dynamics. In all, $N_{1}$ trajectories were generated, and for each trajectory $N_{2}$ realizations of $\mathbf{u}$ and $\mathbf{z}$ were simulated and provided to each of the estimators; these realizations are subsequently referred to as trials. The values used for this simulation are $N_{1}=32, N_{2}=1000, t_{f}=600 \mathrm{~s}$, $f_{\mathbf{u}}=100 \mathrm{~Hz}, f_{k}=1 \mathrm{~Hz}, f_{\mathbf{z}}=10 \mathrm{~Hz}, V_{\text {nominal }}=1 \mathrm{~m} / \mathrm{s}$, $\eta_{V}=0.3 \mathrm{~m} / \mathrm{s}, \eta_{\omega}=0.5 \mathrm{rad} / \mathrm{s}, \sigma_{V}=0.3 \mathrm{~m} / \mathrm{s}, \sigma_{\omega}=0.35 \mathrm{rad} / \mathrm{s}$, $\sigma_{x}=\sigma_{y}=0.03 \mathrm{~m}$, and $\sigma_{\phi}=0.052 \mathrm{rad}$.

A baseline estimator (BL) is also established by propagating the input $\mathbf{u}$ while ignoring the relative measurements $\mathbf{z}$. While a propagation-only approach is typically not practical, it is useful for observing how the various measurement models influence accuracy and consistency. 
Under Review. Submitted to IEEE Control System Magazine on Jan 19, 2017.

\section{Simulation Results}

Figure 4 shows an example result from a single simulation trial for the pseudo-global $(\mathrm{PG})$, stochastic cloning (SC), keyframe-robocentric $(\mathrm{kRC} / \mathrm{kRCi})$, and relative navigation $(\mathrm{RN})$ approaches. In addition to the maximum-likelihood trajectory, the level curve corresponding to the $90 \%$ confidence bound for each estimation approach, derived from the estimator's covariance estimate, is shown. For a given trial any of the estimation approaches could be the most accurate, but the shape and size of the level curves in this figure are representative of the trends observed across all trials.

The following sections present an analysis of the performance of each estimator based on the Monte-Carlo simulation results. The accuracy and consistency of each estimator are examined. In addition, the way in which each estimator handles a global measurement update, if one were to become available, is examined. These results are summarized in Table 2 .

Many of the performance metrics deal with estimation error. Global error for each of the estimation approaches listed in Table 1 is defined as

$$
\begin{aligned}
\mathbf{e}_{\mathrm{BL}} & =\mathbf{x}-\hat{\mathbf{x}}_{\mathrm{BL} g}, \\
\mathbf{e}_{\mathrm{PG}} & =\mathbf{x}-\hat{\mathbf{x}}_{\mathrm{PG} g}, \\
\mathbf{e}_{\mathrm{SC}} & =\mathbf{x}-\hat{\mathbf{x}}_{\mathrm{SC} g}, \\
\mathbf{e}_{\mathrm{kRC}} & =\ominus \mathbf{x}-\hat{\mathbf{x}}_{\mathrm{kRC} g}, \\
\mathbf{e}_{\mathrm{kRCi}} & =\mathbf{x}-\ominus \hat{\mathbf{x}}_{\mathrm{kRCi} g}, \\
\mathbf{e}_{\mathrm{RN}} & =\log \left(\ominus \mathbf{x} \oplus \hat{\mathbf{x}}_{\mathrm{RN} g}\right),
\end{aligned}
$$

where $\hat{\mathbf{x}}_{* g}$ is the global component of the estimated state. Error for a robocentric estimator can be computed either in the body frame $\left(\mathbf{e}_{\mathrm{kRC}}\right)$, or in the inertial frame $\left(\mathbf{e}_{\mathrm{kRCi}}\right)$. The former is relevant when control is computed directly from the body-fixed state estimates. The latter introduces heading error into the inertial position states, but is relevant whenever the estimated states are ultimately utilized in an inertial frame. Error for RN uses the matrix logarithm defined in "The Banana Distribution".

It is important to note that when better input odometry is available, the differences in estimator performance is not as prevalent. The following simulation results should be interpreted in the context of identifying underlying issues and trends, and not a universal judgment on the quality or effectiveness of each estimation approach for a given scenario.

\section{Accuracy}

The accuracy of each estimator is evaluated by averaging the position and absolute heading errors over each trial of each trajectory. Figure 5 shows that for each estimator, as expected, the average error grows unbounded when global measurements are unavailable. However, each estimator loses accuracy at a different rate. By 600 seconds, each estimator has a statistically significant difference in average position and heading error given a 99\% confidence interval, except $\mathrm{kRC}$ and $\mathrm{kRCi}$ position and $\mathrm{kRC}, \mathrm{kRCi}$, and $\mathrm{RN}$ heading. The final error is listed in Table 2. Whether these differences are practically important is application specific.
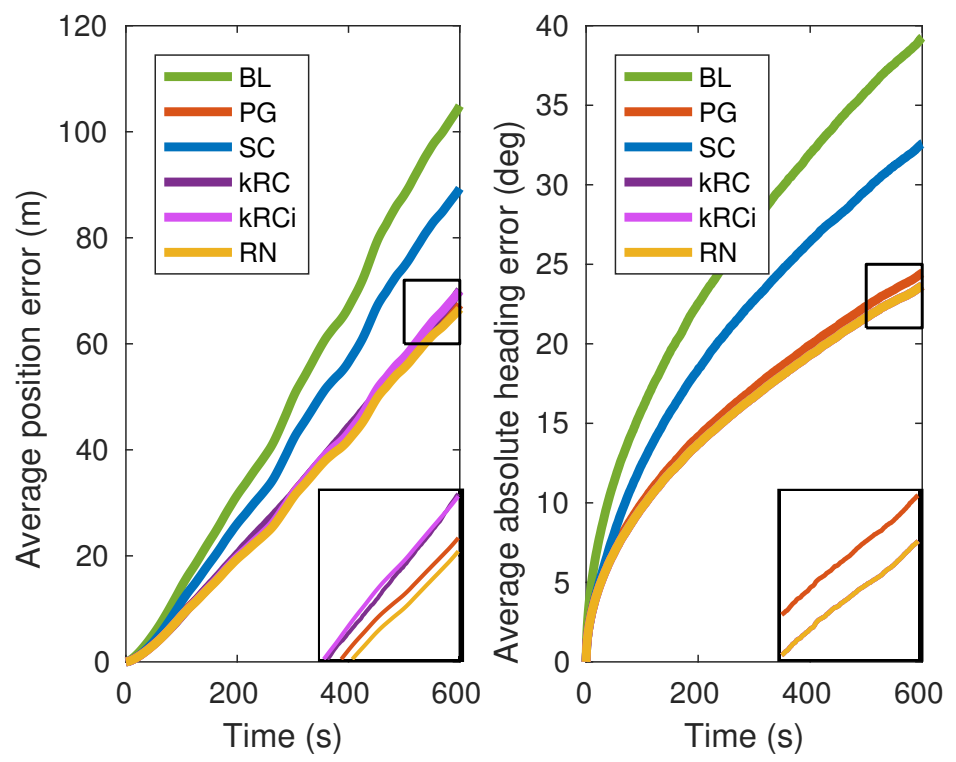

Figure 5: Average position and absolute heading error over each trial on each trajectory. While the estimation error grows unbounded for each approach without global measurements, the error for RN grows at the slowest rate. $\mathrm{kRC}, \mathrm{kRCi}$, and $\mathrm{RN}$ have identical heading error.

$\mathrm{RN}, \mathrm{kRC}$, and PG all have comparatively low error. In their own way, they each apply the relative measurement to a state with a bounded covariance estimate. In contrast, despite using the same relative measurements, SC performs notably worse than the other estimators. One potential explanation for this difference in accuracy is illustrated by the image inset in Figure 4, which shows the jagged nature of the SC trajectory. This jaggedness occurs because the update step for SC sometimes produces unnaturally large corrections, often much larger than the measurement innovation term itself. This phenomenon, first described in [17], occurs when heading uncertainty is allowed to grow unchecked.

\section{Consistency}

A consistent estimator is one whose estimates are unbiased and whose covariance estimate represents well the true underlying uncertainty distribution [8]. Each of these properties are explored in the following sections.

\section{Estimator Bias}

Figure 6 shows, for each estimator, the distribution of errors across all realizations of input and measurement noise for one of the generated trajectories. For all of the estimators except RN, the errors form a banana-like distribution. The expected values of $\mathbf{e}_{\mathrm{BL}}, \mathbf{e}_{\mathrm{PG}}, \mathbf{e}_{\mathrm{SC}}$, and $\mathbf{e}_{\mathrm{kRCi}}$, marked with crosses, are non-zero, indicating a bias that is a direct result of the banana-like distribution. The distribution of $\mathbf{e}_{\mathrm{RN}}$ in Figure 6f, on the other hand, is not banana shaped because the matrix logarithm unwarps the distribution. As a result, the expected value of $\mathbf{e}_{\mathrm{RN}}$ is approximately zero. As an addendum to the discussion in "The Banana Distribution", the fact that Figure $6 \mathrm{f}$ appears approximately elliptical suggests that a Gaussian covariance parameterization in exponential 
Under Review. Submitted to IEEE Control System Magazine on Jan 19, 2017.

Table 2: Summary comparison of the estimation approaches. RN exhibits improved performance in terms on of average position error, average absolute heading error, estimation bias, and average normalized estimation error squared (NEES). When a global measurement $\mathbf{z}_{g}$ is eventually applied at $t_{f}$, the global estimation approaches either experience a large state jump or the measurement is rejected.

\begin{tabular}{lcccccc}
\hline & $\mathrm{BL}$ & $\mathrm{PG}$ & $\mathrm{SC}$ & $\mathrm{kRC}$ & $\mathrm{kRCi}$ & $\mathrm{RN}$ \\
\hline Average position error at $t_{f}(\mathrm{~m})$ & 104.9 & 67.4 & 89.3 & 70.1 & 69.9 & 66.6 \\
Average absolute heading error at $t_{f}(\mathrm{deg})$ & 39.3 & 24.5 & 32.6 & 23.7 & 23.7 & 23.7 \\
Number of biased trajectories at $99 \%$ level & $31 / 32$ & $31 / 32$ & $31 / 32$ & $4 / 32$ & $32 / 32$ & $0 / 32$ \\
Average NEES at $t_{f}$ & 10.2 & $3.52 \mathrm{e} 7$ & 175 & 7.77 & 7.89 & 3.09 \\
Average position update given $\mathbf{z}_{g}(\mathrm{~m})$ & 104.2 & $7.2 \mathrm{e}-4$ & 70.3 & 69.0 & - & - \\
Average absolute heading update after $\mathbf{z}_{g}(\mathrm{deg})$ & 31.4 & 0.02 & 10.5 & 19.5 & - & - \\
\hline
\end{tabular}

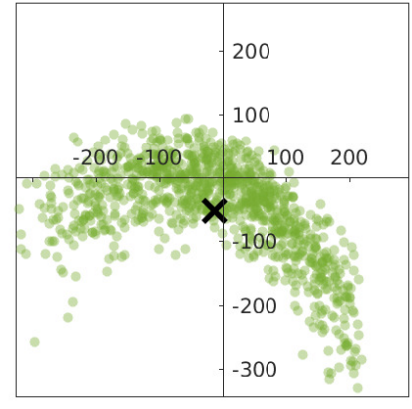

(a) $\mathbf{e}_{\mathrm{BL}}$

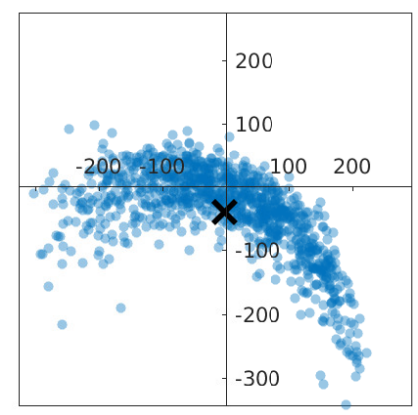

(c) $\mathbf{e}_{\mathrm{SC}}$

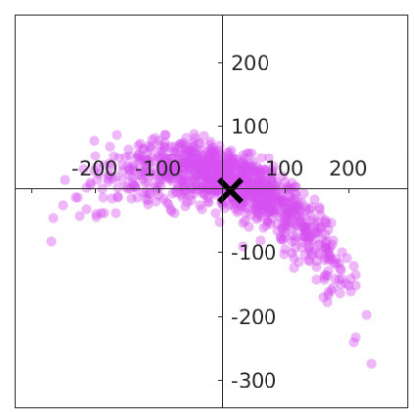

(e) $\mathbf{e}_{\mathrm{kRCi}}$

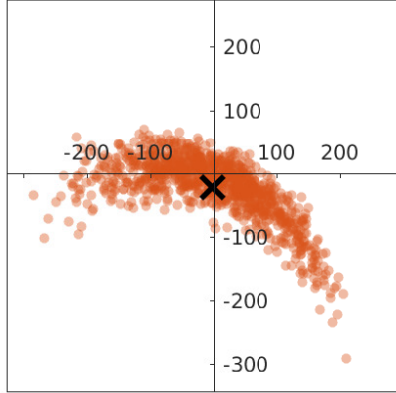

(b) e ePG

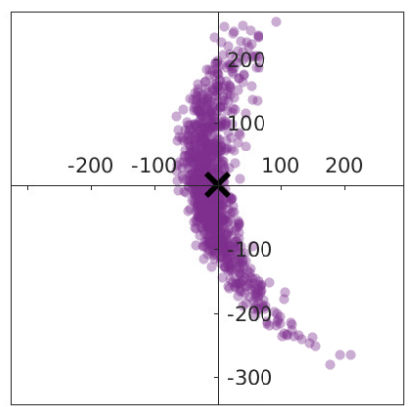

(d) $\mathbf{e}_{\mathrm{kRC}}$

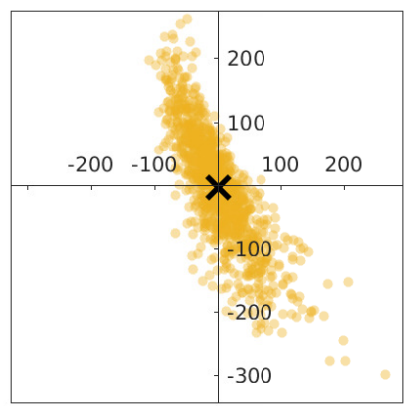

(f) $\mathbf{e}_{\mathrm{RN}}$

Figure 6: Estimation error for one of the trajectories. The cross indicates the mean error. $\mathrm{BL}, \mathrm{PG}, \mathrm{SC}$, and $\mathrm{kRCi}$ are biased, while $\mathrm{kRC}$ and $\mathrm{RN}$ are not. Units are in meters

coordinates is appropriate for the $\mathrm{RN}$ pose graph representation.

To investigate bias more precisely, the Hotelling $T^{2}$ statistic is computed for each estimator and each trajectory. The statistic is defined as

$$
T^{2}=N_{2} \overline{\mathbf{e}}^{\top} \mathbf{S}^{-1} \overline{\mathbf{e}},
$$

where $\overline{\mathbf{e}}$ and $\mathbf{S}$ are the sample mean and covariance, and is distributed according to

$$
T^{2} \sim \frac{p\left(N_{2}-1\right)}{N_{2}-p} F_{p, N_{2}-p},
$$

where $p=3$ is the dimensionality of the state vector and $F$ is the $F$-distribution with the indicated degrees of freedom. Table 2 indicates the number of trajectories for each estimator that are statistically biased at a 99 percent confidence level. $\mathrm{RN}$ is the only estimation approach that can be considered unbiased for every trajectory at this level. kRC is usually not biased while working in the body-centered frame, but is always biased when represented in an inertial frame $(\mathrm{kRCi})$.

\section{Estimator Uncertainty}

Having an accurate covariance estimate is important for proper sensor fusion [8]. In addition, many higher-level algorithms, such as path planning, rely on accurate uncertainty estimates.

Figure 4 shows the typical sizes and shapes of the uncertainty estimates for each approach. The covariance of the PG approach remains on the same order as the relative measurement uncertainty $\mathbf{R}$, which is too small to be seen in Figure 4 . The covariance of the SC approach does not grow sufficiently because the measurement model artificially introduces extra information. Additional steps can reduce this effect [18], but the root cause - unobservability - is avoided altogether by the $\mathrm{RN}$ approach. Because $\mathrm{kRC}$ predominately works in an observable, local frame, the uncertainty estimate grows at the appropriate rate [19], but like PG, SC, and BL the covariance representation only supports an elliptical confidence bound. The banana-like confidence bound of RN properly represents the true uncertainty distribution.

The degree to which the underlying uncertainty is wellmodeled is evaluated using the normalized estimation error squared (NEES), defined at each time step $t$ as

$$
\epsilon_{t}=\mathbf{e}_{t}^{\top} \mathbf{P}_{t}^{-1} \mathbf{e}_{t} .
$$


Under Review. Submitted to IEEE Control System Magazine on Jan 19, 2017.
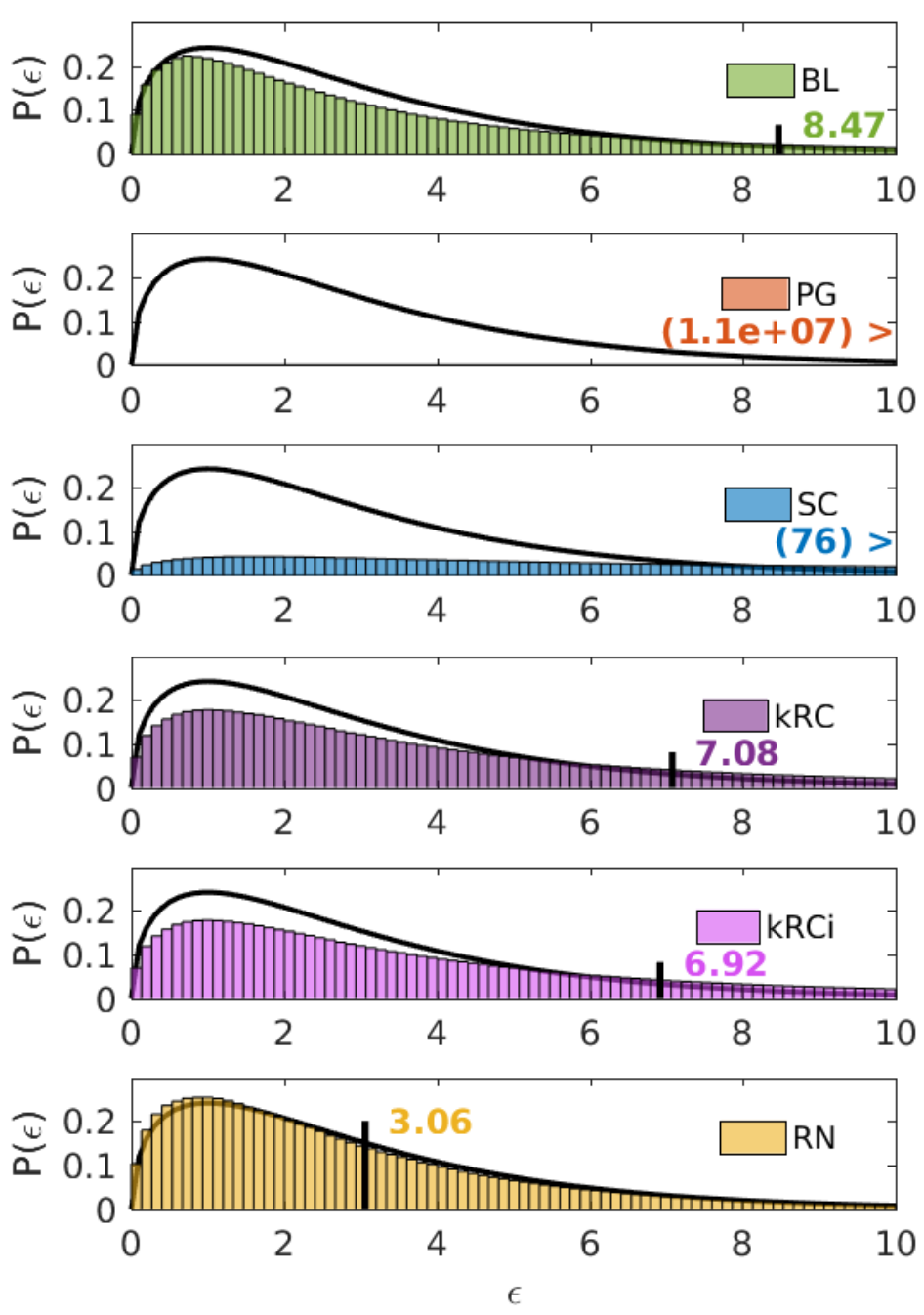

Figure 7: Normalized estimation error squared (NEES) probability density function (pdf) for each estimation approach with the ideal $\chi_{3}^{2}$ pdf overlaid. $\epsilon$ is calculated once per second over each trial of each trajectory. RN appears globally consistent while the other approaches are overconfident. The distribution mean is labeled, where the ideal mean is 3 .

This metric, commonly referred to as the Mahalanobis distance, weights the deviations in each state based on the inverse of the associated uncertainty. When $\mathbf{P}$ is the true covariance, NEES has the property

$$
\epsilon \sim \chi_{p}^{2}, \quad E[\epsilon]=p,
$$

where $p=3$ is dimensionality of the state vector.

Figure 7 presents the observed probability distribution function (pdf) of $\epsilon$ for each approach. $\epsilon$ was calculated once per second over each trial of each trajectory. The ideal $\chi_{3}^{2}$ pdf is overlaid and the expected value is labeled. When the observed distribution is more heavily weighted on the left than the ideal $\chi_{3}^{2}$ distribution, the filter is said to be conservative, indicating the covariance is too large. Conversely, when the observed distribution has a longer or fatter tail to the right than the ideal, the filter is optimistic [17] or overconfident. It is usually better for an estimator to be conservative.

From Figure 7 we see RN appears to be globally consistent in spite of prolonged GPS-dropout and substantial heading

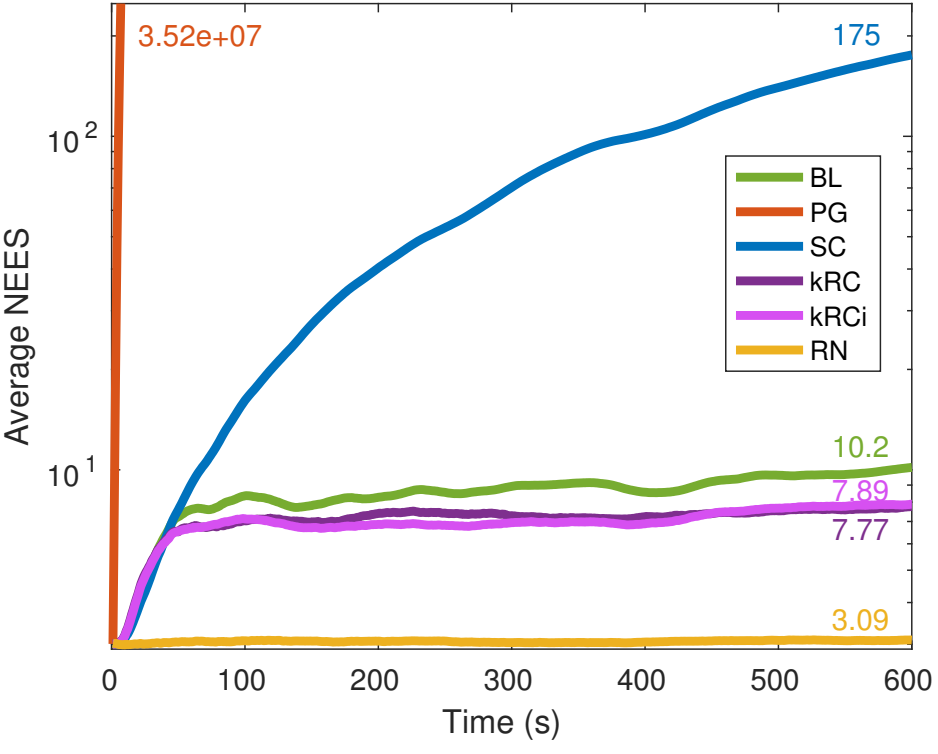

Figure 8: Average normalized estimation error squared (NEES) over all trials and all trajectories as a function of time. The expected value for a consistent estimator should be 3. RN begins and stays near the ideal while the other approaches lose consistency from the start.

uncertainty. PG does not provide an accurate global uncertainty estimate where the smallest $\epsilon_{\mathrm{PG}}$ is 320 . Only occasionally does SC provide a reasonable global covariance estimate given these circumstances. $\mathrm{kRC}, \mathrm{kRCi}$, and $\mathrm{BL}$ follow the general trend but experience an abnormally high number of large NEES estimates. For example, given the particularly erroneous inputs illustrated in Figure $4, \epsilon_{\mathrm{BL}}=279$, $\epsilon_{\mathrm{PG}}=2.7 \times 10^{8}, \epsilon_{\mathrm{SC}}=1275, \epsilon_{\mathrm{kRC}}=39.8, \epsilon_{\mathrm{kRCi}}=67.9$, and $\epsilon_{\mathrm{RN}}=6.03$ at time $t_{f}$.

Because we are exploring how estimation approaches break down, it is insightful to see how the average NEES changes over time. In Figure 8 we see RN begins and stays approximately equal to the ideal value of three. PG almost immediately loses consistency. Interestingly, the consistency of BL, $\mathrm{SC}, \mathrm{kRC}$, and $\mathrm{kRCi}$ all degrade at the same rate for the first 50 seconds. At this point, $\mathrm{SC}$ continues to degrade while the others approximately settle out. Plots similar to Figure 8 are common in the SLAM literature, though typically only $20-50$ trials are averaged $[17,29,30]$. They similarly show that without global information such as loop closures consistency is lost, even when using an iterative EKF, unscented $\mathrm{KF}$, or ideal Jacobians evaluated at the true unknown state. $\mathrm{RN}$ avoids this issue completely by working in an observable frame.

\section{Eventual Global Update}

If a global measurement such as GPS becomes available, it is useful to incorporate that information into the state estimate. After a prolonged period of GPS dropout, the discrepancy between the true and estimated global position is likely to be large due to drift in the estimates. When an estimator's uncertainty is also large it will readily accept the measurement, causing a large state jump that could potentially produce a large spike in control effort. This is particularly 
Under Review. Submitted to IEEE Control System Magazine on Jan 19, 2017.

troubling when GPS is degraded; even when the global information is accurate, however, directly fusing the information using a Kalman filter update is known to degrade both the estimation and control $[1,31]$. The extent to which each approach is affected by a delayed global update is examined in the following extension to the simulation.

For each trial a global position measurement $\mathbf{z}_{g}$ is generated, where $\mathbf{z}_{g} \sim \mathcal{N}\left(\mathbf{x}_{t_{f}}, R_{\mathbf{z}_{g}}\right)$ and $\mathbf{R}_{\mathbf{z}_{g}}=\operatorname{diag}(25,25)$. Using (2)-(4), the state update is computed as

$$
\Delta=\mathbf{K}\left(\mathbf{z}_{g}-h(\hat{\mathbf{x}})\right) .
$$

While the measurement only includes position information, the heading estimate is updated through the cross-correlation covariance terms. Table 2 lists the average magnitude of the position and heading updates. It is informative to note the magnitude of the average update as well as its relative size to the average error. From (3) it is clear that if $\mathbf{P}$ is substantially smaller than $\mathbf{R}$, the Kalman gain approaches zero and the measurement innovation is largely rejected, as demonstrated with PG. Conversely, when $\mathbf{P}$ is substantially larger than $\mathbf{R}$, the Kalman gain approaches identity and the measurement innovation is readily accepted, causing a large state jump as demonstrated by BL and kRC. SC only partially accepts the global update. None of these scenarios are conducive to robust MAV navigation and control.

Several approaches have been presented to work around these issues, such as simultaneously tracking a GPS-corrected and odometry-only global trajectory $[1,31]$, or using a series of measurement gates [32]. Other approaches refrain from incorporating GPS into the filter at all, opting instead to incorporate GPS exclusively using a pose graph [33].

Because RN only estimates a relative state, directly fusing an eventual global measurement is not an option. Rather, these measurements are incorporated using a pose-graph map as described in [21]. This formulation completely avoids the problem of large jumps in the filter states that are used for control. In addition, it makes it possible to identify erroneous measurements and completely eliminate their effect on the global state estimate at any time as more information is received.

\section{Summary of Implications}

Implications of the simulation results are summarized in Figures 9 and 10. Rather than showing data flow, these diagrams illustrate consequences, where implications are represented as connecting arrows. The blue boxes, comprising prolonged GPS-dropout, heading uncertainty, intermittent and erroneous global measurements, as well as loop-closure constraints, represent the non-ideal yet realistic inputs to the system. Green boxes highlight the deficiencies that inevitably result.

The simulation results are summarized by discussing paths through Figure 9, proceeding from top to bottom and from left to right. During prolonged GPS dropout and in the presence of heading uncertainty, the global position and heading state will inevitably drift. This drift will induce an underlying probability distribution that is banana-shaped, which is not well modeled by a Gaussian in Cartesian coordinates.
Further, some approaches tend to introduce undue information into the filter, artificially constraining the uncertainty magnitude. Eventually, this leads to inconsistent uncertainty estimates, causing measurements to be fused sub-optimally. When filters properly acknowledge that the state is unobservable, the uncertainty will grow without bound. A large uncertainty also causes the system to strongly trust eventual global measurements, whether they be degraded or not, often leading to large state updates. Sudden jumps in state lead to degraded position control, impacting MAV reliability. Finally, many approaches attempt to reduce drift by identifying when the vehicle returns to a previously visited location. Incorporating loop closure constraints through non-linear optimization techniques also results in large state updates and either presents non-negligible delays, stresses the platform's size, weight and power (SWaP) constraints, or assumes an uninterrupted network connection.

A wide variety of techniques are used in practice to address the issues presented in Figure 9, particularly for handling large state updates. It should be noted, however, that the root of each issue stems from working with respect to an unobservable coordinate frame. Figure 10 illustrates how decoupling the system into relative and global frames allows for optimal navigation within the local frame itself, avoiding many of these issues entirely. Because relative measurements directly update the relative state, the state covariance represents the underlying uncertainty well, leading to optimal sensor fusion. While the non-ideal inputs and inevitable deficiencies may hamper the completion of a global mission, they need not degrade the stability of the system. For example, in the worst case, a large, erroneous global update results in an incorrect relative goal.

\section{Hardware Results}

The simulation results constitute the primary contributions of this article, showing that global keyframe filters break down more readily than relative navigation during prolonged GPS dropout. To supplement these contributions, this section presents the estimation performance of each method when implemented on MAV hardware. This section is not intended to thoroughly describe all MAV implementation details, but rather to demonstrate that the performance of full-state estimators running on actual hardware parallels the results seen in simulation. Complete details on the relative navigation implementation used for these results, along with a presentation of more extensive flight-test results, are given in [21].

Reference [20] includes the details necessary to implement a relative state estimator for a MAV, including the vehicle dynamics and measurement models. The estimator implementations of each approach for the results in this article are all based on the error-state, multiplicative extended Kalman filter described in [20]. The changes needed to adapt this filter to each of the different estimation approaches were minimal, requiring modifications to less than ten lines of code for each approach. The vehicle's state includes position, velocity, attitude parameterized with a quaternion, and gyroscope and accelerometer biases. Inputs were body-fixed accelerometer and gyroscope measurements at $100 \mathrm{~Hz}$ from a calibrated 
Under Review. Submitted to IEEE Control System Magazine on Jan 19, 2017.

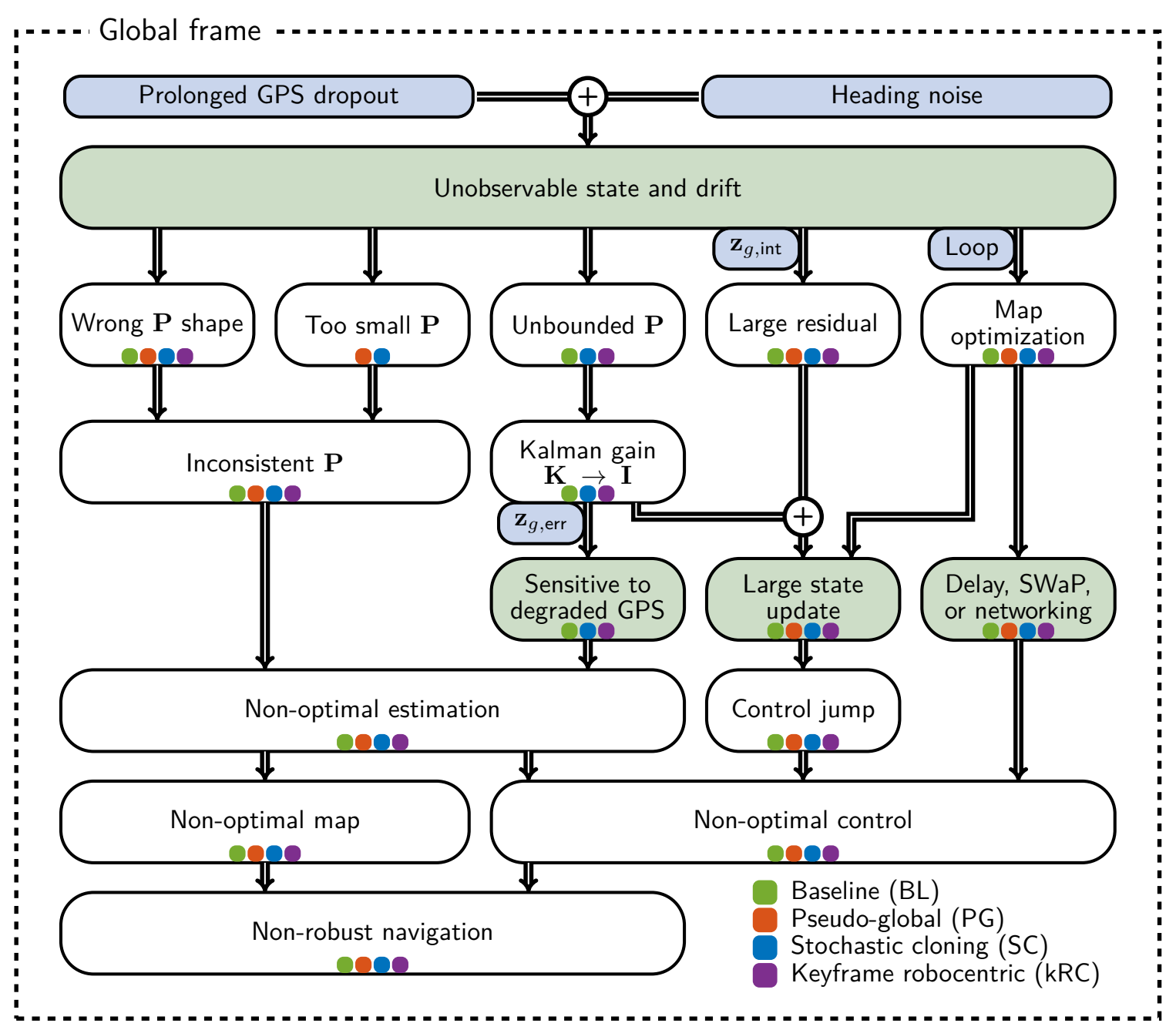

Figure 9: Diagram summarizing the implications that prolonged GPS dropout and heading noise have when working in a global coordinate frame. Arrows indicate implications, blue boxes highlight non-ideal yet realistic inputs to the system, and green boxes highlight inevitable deficiencies. Ultimately global drift will induce an inconsistent and/or unbounded state uncertainty estimate $\mathbf{P}$ which leads to non-robust navigation. For each approach, the relevant consequences are labeled according to the legend.

MicroStrain 3DM-GX3-15 IMU. A MaxBotix MB1242 ultrasonic altimeter provided height-above-ground measurements, while visual odometry updates were obtained at $15 \mathrm{~Hz}$ using the algorithm from [34] with an ASUS Xtion Pro Live RGB-D camera.

A user provided velocity commands to navigate the multirotor around the perimeter of the building shown in Figure 11. The flight lasted nine minutes and traversed 320 meters. The RN estimator provided the necessary onboard state estimates for feedback control. Afterwards, time-stamped measurements were provided to each estimator resulting in the trajectory and covariance estimates shown in Figure 11.

As shown in Figure 11, the flight-test produced similar results to those seen in simulation. While a single trial cannot be used to make claims about the relative accuracy of the different approaches, all of the approaches produced reasonably accurate results with errors on a similar order of magnitude. Like in the simulation results, PG produced an extremely overconfident uncertainty bound, while SC produced a somewhat better, but still overconfident, uncertainty estimate. The RN approach produced a banana-shaped uncer- tainty bound that includes the true pose of the vehicle.

Because conventional multirotor dynamics assume an inertial reference frame, the robocentric displacement vector $\hat{\mathbf{x}}^{\Delta}$ in Figure 2c cannot be propagated directly. Instead, following [35] $\mathrm{kRC}$ was implemented using vehicle dynamics expressed with respect to the body, also described in [20]. Because these position dynamics do not depend on the current attitude, the EKF has no mechanism to properly increase position uncertainty due to heading uncertainty. As a result, the $\mathrm{kRC}$ confidence bound remained small.

To provide a more direct comparison to the body-fixed implementation of $\mathrm{kRC}$, relative navigation was additionally implemented using the body-fixed dynamics (bRN) presented in [20]. In contrast to $\mathrm{kRC}$, the coupling between position and heading uncertainty at the keyframe level is negligible. As a result, when the global state and uncertainty are reconstructed using a sequence of many relative states, as described in "The Banana Distribution", a reasonable banana-shaped confidence bound results. A more thorough investigation into the consistency of bRN remains as future work. 
Under Review. Submitted to IEEE Control System Magazine on Jan 19, 2017.

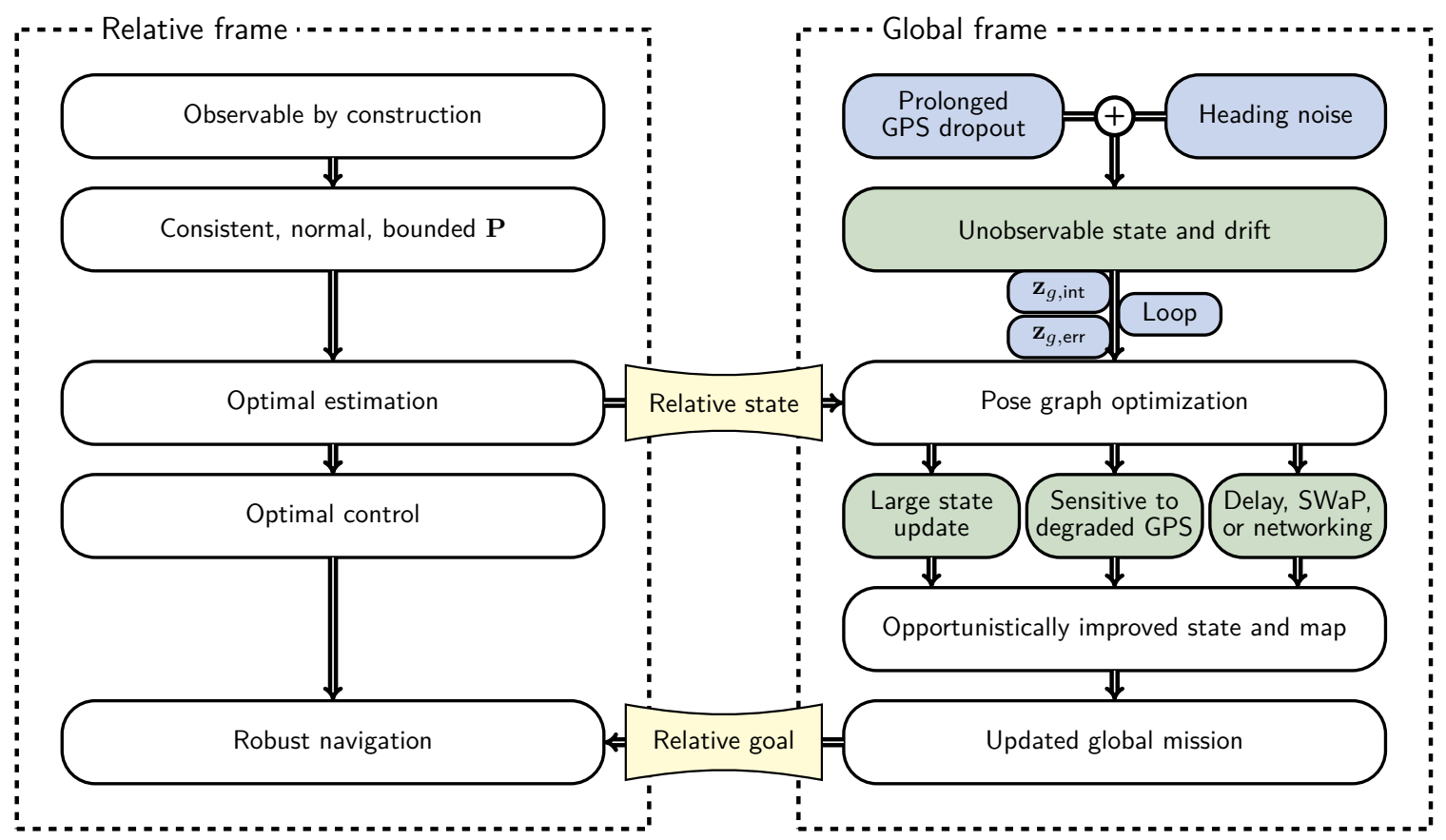

Figure 10: Diagram summarizing the implications that prolonged GPS dropout and heading noise has when global and relative frames are decoupled (i.e. relative navigation). Note that unlike the global estimation techniques described in Figure 9, the non-ideal inputs (blue) and inevitable deficiencies (green) are specific to the global frame and do not affect the robustness of relative navigation.

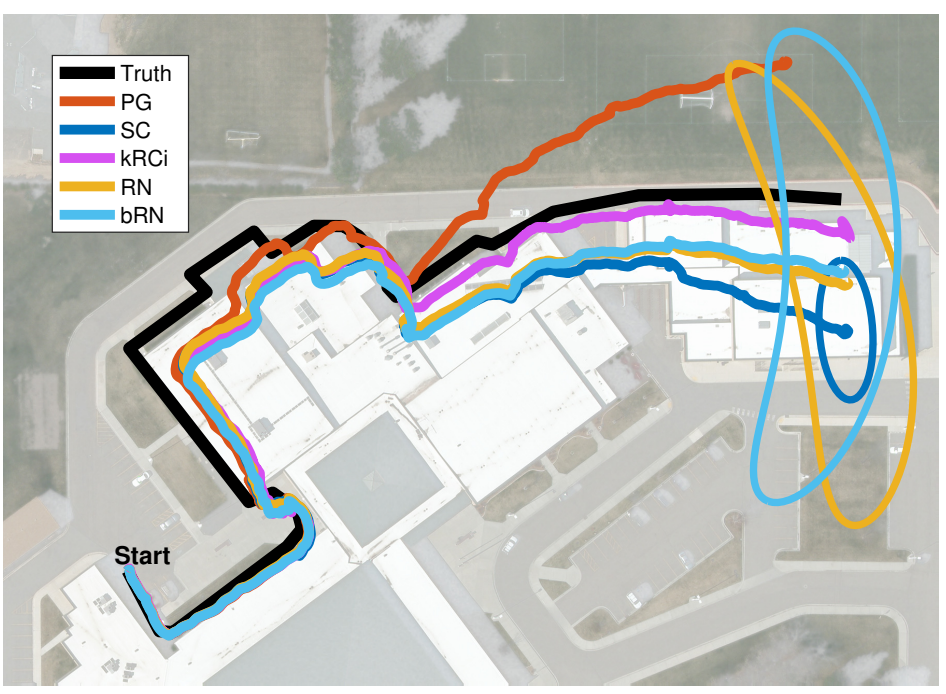

\section{Conclusions}

Global drift is inevitable when MAVs fly in GPS-denied environments because the global position and heading states are unobservable when only relative measurements are available. While most navigation frameworks estimate the global state directly despite this unobservability, relative navigation maintains local observability by estimating the vehicle states with respect to a local frame. As demonstrated in this article, estimating and controlling with respect to a local frame produces more consistent global position estimates and avoids many of the consistency and stability issues common to existing global approaches. Relative navigation also provides a framework for robustly incorporating intermittent global information. Moving from a global to a relative estimation approach is a fairly small adjustment conceptually and in terms of implementation, but yields significant advantages that can benefit systems that currently use a global estimation approach.

\section{Acknowledgments}

90 percent confidence bounds are drawn. A body-fixed relative navigation (bRN) approach was also included. While $\mathrm{kRCi}$ had the lowest error, the overall accuracy of an approach should only be evaluated after many trials, as done in Figure 5. However, the confidence bounds are typical; only $\mathrm{RN}$ and bRN include the true state. BL quickly degraded due to significant IMU noise and was excluded from the figure. (Background image courtesy of U.S. Geological Survey)

This work has been funded by the Center for Unmanned Aircraft Systems (C-UAS), a National Science Foundation Industry/University Cooperative Research Center (I/UCRC) under NSF award Numbers IIP-1161036 and CNS-1650547, along with significant contributions from C-UAS industry members. This work was also supported in part by Air Force Research Laboratory Science and Technology (AFRL S\&T) sponsorship. Daniel Koch was supported by the Department of Defense (DoD) through the National Defense Science \& Engineering Graduate Fellowship (NDSEG) Program. The authors would like to thank Kevin Brink of the Air Force Re- 
search Laboratory Munitions Directorate (AFRL/RW) for his support of this project and for his valuable insights. 


\section{References}

[1] S. Shen, Y. Mulgaonkar, N. Michael, and V. Kumar, "Multi-sensor fusion for robust autonomous flight in indoor and outdoor environments with a rotorcraft MAV," in Proc. IEEE Int. Conf. Robotics and Automation, pp. 4974-4981, 2014.

[2] R. C. Leishman and T. W. McLain, "Multiplicative extended Kalman filter for relative rotorcraft navigation," J. Aerospace Inform. Syst., vol. 12, no. 12, pp. 728-744, 2014.

[3] S. Leutenegger, S. Lynen, M. Bosse, R. Siegwart, and P. Furgale, "Keyframe-based visual-inertial odometry using nonlinear optimization," Int. J. Robotics Research, vol. 34, no. 3, pp. 314-334, 2015.

[4] A. Bachrach, S. Prentice, and N. Roy, "RANGE-Robust autonomous navigation in GPS-denied environments," $J$. Field Robotics, vol. 28, no. 5, pp. 644-666, 2011.

[5] S. Weiss, M. W. Achtelik, S. Lynen, M. Chli, and R. Siegwart, "Real-time onboard visual-inertial state estimation and self-calibration of MAVs in unknown environments," in Proc. IEEE Int. Conf. Robotics and Automation, pp. 957-964, 2012.

[6] E. Jones, A. Vedaldi, and S. Soatto, "Inertial structure from motion with autocalibration," in ICCV Workshop on Dynamical Vision, 2007.

[7] A. Martinelli, "Vision and IMU data fusion : Closedform solutions," IEEE Transactions on Robotics, vol. 28, no. 1, pp. 44-60, 2012.

[8] Y. Bar-Shalom, T. Kirubarajan, and X.-R. Li, Estimation with Applications to Tracking and Navigation. New York: John Wiley \& Sons, Inc., 2002.

[9] D. Scaramuzza and F. Fraundorfer, "Visual odometry [tutorial]," IEEE Robot. Autom. Mag., vol. 18, no. 4, pp. 80-92, 2011.

[10] F. Fraundorfer and D. Scaramuzza, "Visual odometry: Part II: Matching, robustness, optimization, and applications," IEEE Robot. Autom. Mag., vol. 19, no. 2, pp. 78 90, 2012.

[11] J.-S. Gutmann and C. Schlegel, "AMOS: Comparison of scan matching approaches for self-localization in indoor environments," in Proc. 1st Euromicro Workshop on Advanced Mobile Robots, pp. 61-67, 1996.

[12] R. Smith, M. Self, and P. Cheeseman, "A stochastic map for uncertain spatial relationships," in Proc. 4th Int. Symp. Robotics Research, pp. 467-474, 1988.

[13] D. Scaramuzza, M. C. Achtelik, L. Doitsidis, F. Friedrich, E. Kosmatopoulos, A. Martinelli, M. W. Achtelik, M. Chli, S. Chatzichristofis, L. Kneip, D. Gurdan, L. Heng, G. H. Lee, S. Lynen, M. Pollefeys, A. Renzaglia, R. Siegward, J. C. Stumpf, P. Tanskanen, C. Troiani, S. Weiss, and L. Meier, "Vision-controlled micro flying robots: from system design to autonomous navigation and mapping in GPS-denied environments," IEEE Robot. Autom. Mag., vol. 21, no. 3, pp. 26-40, 2014.

[14] S. Weiss and R. Siegwart, "Real-time metric state estimation for modular vision-inertial systems," in Proc. IEEE Int. Conf. Robotics and Automation, pp. 45314537, 2011.

[15] B. D. Hoffman, E. T. Baumgartner, T. L. Huntsberger, and P. S. Schenker, "Improved rover state estimation in challenging terrain," Autonomous Robots, vol. 6, no. 2, pp. 113-130, 1999

[16] S. I. Roumeliotis and J. Burdick, "Stochastic cloning: a generalized framework for processing relative state measurements," in Proc. IEEE Int. Conf. Robotics and Automation, pp. 1788-1795, 2002.

[17] T. Bailey, J. Nieto, J. Guivant, M. Stevens, and E. Nebot, "Consistency of the EKF-SLAM algorithm," in Proc. IEEE Int. Conf. Intelligent Robots and Syst., pp. 3562 $3568,2006$.

[18] G. P. Huang, A. I. Mourikis, and S. I. Roumeliotis, "Observability-based rules for designing consistent EKF SLAM estimators," Int. J. Robotics Research, vol. 29, no. 5 , pp. 502-528, 2010 .

[19] J. A. Castellanos, R. Martinez-Cantin, J. D. Tardós, and J. Neira, "Robocentric map joining: Improving the consistency of EKF-SLAM," Robotics and Autonomous Syst., vol. 55, no. 1, pp. 21-29, 2007.

[20] D. P. Koch, D. O. Wheeler, R. W. Beard, T. W. McLain, and K. M. Brink, "Relative multiplicative extended Kalman filter for observable GPS-denied navigation," 2017. Available at http://scholarsarchive. byu. edu/facpub/1963/.

[21] D. O. Wheeler, D. P. Koch, J. S. Jackson, G. J. Ellingson, P. W. Nyholm, T. W. McLain, and R. W. Beard, "Relative navigation of autonomous GPS-degraded micro air vehicles," 2017. Available at http://scholarsarchive. byu. edu/facpub/1962/.

[22] R. C. Leishman, T. W. McLain, and R. W. Beard, "Relative navigation approach for vision-based aerial GPS-denied navigation," J. Intelligent and Robotic Syst., vol. 74, no. 1, pp. 97-111, 2014.

[23] T. D. Barfoot and P. T. Furgale, "Associating uncertainty with three-dimensional poses for use in estimation problems," IEEE Trans. Robot., vol. 30, no. 3, pp. 679693, 2014.

[24] K. S. Chong and L. Kleeman, "Feature-based mapping in real, large scale environments using an ultrasonic array," Int. J. Robotics Research, vol. 18, no. 1, pp. 3-19, 1999.

[25] B. Kim, M. Kaess, L. Fletcher, J. Leonard, A. Bachrach, N. Roy, and S. Teller, "Multiple relative pose graphs for robust cooperative mapping," in Proc. IEEE Int. Conf. Robotics and Automation, pp. 3185-3192, 2010. 
[26] G. Grisetti, R. Kummerle, C. Stachniss, and W. Burgard, "A tutorial on graph-based SLAM," IEEE Intell. Transp. Syst. Mag., vol. 2, no. 4, pp. 31-43, 2010.

[27] R. Kummerle, G. Grisetti, H. Strasdat, K. Konolige, and W. Burgard, "g2o: A general framework for graph optimization," in Proc. IEEE Int. Conf. Robotics and Automation, pp. 3607-3613, 2011.

[28] M. Kaess, A. Ranganathan, and F. Dellaert, "iSAM: Incremental smoothing and mapping," IEEE Trans. Robot., vol. 24, no. 6, pp. 1365-1378, 2008.

[29] G. P. Huang, A. I. Mourikis, and S. I. Roumeliotis, "Analysis and improvement of the consistency of extended Kalman filter based SLAM," in Proc. IEEE Int. Conf. Robotics and Automation, pp. 473-479, 2008.

[30] J. Sola, "Consistency of the monocular EKF-SLAM algorithm for three different landmark parametrizations," in Proc. IEEE Int. Conf. Robotics and Automation, pp. 3513-3518, 2010.

[31] S. A. Scherer, S. Yang, and A. Zell, "DCTAM: Driftcorrected tracking and mapping for autonomous micro aerial vehicles," in Proc. Int. Conf. Unmanned Aircraft Syst., pp. 1094-1101, 2015.

[32] A. Chambers, S. Scherer, L. Yoder, S. Jain, S. Nuske, and S. Singh, "Robust multi-sensor fusion for micro aerial vehicle navigation in GPS-degraded/denied environments," pp. 1892-1899, June 2014.

[33] A. Chambers, S. Achar, S. Nuske, J. Rehder, B. Kitt, L. Chamberlain, J. Haines, S. Scherer, and S. Singh, "Perception for a river mapping robot," in Proc. IEEE/RSJ Int. Conf. Intelligent Robots and Syst., pp. 227-234, 2011.

[34] J. Zhang, M. Kaess, and S. Singh, "Real-time depth enhanced monocular odometry," in Proc. IEEE/RSJ Int. Conf. Intelligent Robots and Syst., pp. 4973-4980, 2014.

[35] M. Bloesch, S. Omari, M. Hutter, and R. Siegwart, "Robust visual inertial odometry using a direct EKF-based approach," in Proc. IEEE Int. Conf. Intelligent Robots and Syst., pp. 298-304, 2015.

[36] H. Durrant-Whyte and T. Bailey, "Simultaneous localization and mapping: Part I," IEEE Robot. Autom. Mag., vol. 13, no. 2, pp. 99-110, 2006.

[37] T. Bailey and H. Durrant-Whyte, "Simultaneous localization and mapping (SLAM): Part II," IEEE Robot. Autom. Mag., vol. 13, no. 3, pp. 108-117, 2006.

[38] M. Dissanayake, P. Newmann, S. Clark, H. F. DurrantWhite, and M. Corsba, "A solution to the simultaneous localisation and map building problem," IEEE Trans. Robot. Autom., vol. 17, no. 3, pp. 229-257, 2001.

[39] S. Thrun, W. Burgard, and D. Fox, Probabilistic Robotics. MIT Press, 2005.
[40] G. Chowdhary, E. N. Johnson, D. Magree, A. Wu, and A. Shein, "GPS-denied indoor and outdoor monocular vision-aided navigation and control of unmanned aircraft," vol. 30, pp. 415-438, May 2013.

[41] C. Forster, L. Carlone, F. Dellaert, and D. Scaramuzza, "IMU preintegration on manifold for efficient visualinertial maximum-a-posteriori estimation," in Robotics: Science and Systems, 2015.

[42] S. Thrun, W. Burgard, and D. Fox, "A real-time algorithm for mobile robot mapping with applications to multi-robot and 3D mapping," in Proc. IEEE Int. Conf. Robotics and Automation, pp. 321-328, 2000.

[43] A. W. Long, K. C. Wolfe, M. J. Mashner, and G. S. Chirikjian, "The banana distribution is Gaussian: A localization study with exponential coordinates," in Proc. Robotics: Sci. and Syst., 2012. 


\section{Sidebar: Article Summary}

State estimation for micro air vehicles (MAVs) often depends heavily on reliable global measurements such as GPS. When global measurements are unavailable, additional sensors, such as cameras or laser scanners, are commonly used to provide measurements of the MAV's translation and rotation relative to a previously observed keyframe image or scan. With the use of only relative sensors, however, the global position and heading of the vehicle are unobservable and cannot be reliably reconstructed. Many existing approaches work with respect to a global reference frame, resulting in a loss of state observability. This article highlights that unobservability leads to inconsistency and a loss of optimality, which reduces estimation accuracy and robustness of the navigation solution. Relative navigation is presented as an alternative approach that maintains observability by always working with respect to a local coordinate frame. While still subject to global drift, relative navigation is shown through rigorous simulation and hardware validation to produce accurate and consistent state estimates when other approaches break down. By subtly restructuring the state estimation problem to a relative framework, many of the pitfalls prevalent in GPS-denied MAV navigation systems are inherently mitigated. 


\section{Sidebar: Connection to Simultaneous Localization and Mapping}

When a vehicle enters an unknown, GPS-denied environment, it must estimate both its state and the locally observed environment. The simultaneous localization and mapping (SLAM) problem solves for the most probable vehicle trajectory and landmark locations, given a time history of inputs and relative measurements to the local environment [36,37]. Often, simplifying assumptions are made to reduce the computational burden on resource-constrained platforms. While full-SLAM solves for the most likely vehicle trajectory, onlineSLAM, such as EKF-SLAM [38], solves only for the current state of the vehicle and nearby features or landmarks [39]. Keyframe filters further simplify the estimation problem by tracking a single keyframe rather than many individual landmarks.

MAV state estimators are generally limited to solving some simplified form of the SLAM problem due to size, weight, and power (SWaP) limitations specific to each platform. Some MAV navigation solutions are derived from the EKF-SLAM framework $[35,40]$, while others avoid filtering techniques altogether, electing to solve for the MAV's pose using non-linear optimization techniques such as factor graphs [41]. Yet many others choose to use keyframe-based approaches for their reduced computational complexity $[1,3,4,16,22]$. Each approach works towards solving similar problems but with different assumptions and different formulations.

Another prominent research emphasis for SLAM is maintaining consistency. While traditional SLAM approaches estimate the vehicle and landmark locations with respect to a global coordinate frame, it has been shown that such parameterizations lose consistency as heading uncertainty increases [17]. As such, an increasing number of relative SLAM implementations are being published. Robocentric approaches estimate the pose of landmarks and the global origin with respect to the vehicle's current position and attitude [19]. Relative submaps estimate the state of the vehicle and landmarks with respect to a local inertial coordinate frame [24,25]. These submaps are subsequently fused and form a more consistent global estimate.

In summary, keyframe-based filters are common for MAV state estimation because of the reduced computational burden, while relative formulations are common within the SLAM literature to improve consistency. This article presents a relative keyframe filter, which we call relative navigation, as the logical method to improve consistency of global keyframe approaches. The purpose of this article is not to compare the performance of keyframe-based MAV navigation approaches to other SLAM techniques, but rather to rigorously compare relative navigation to state-of-the-art global keyframe filters. These ideas are illustrated in Figure 12.

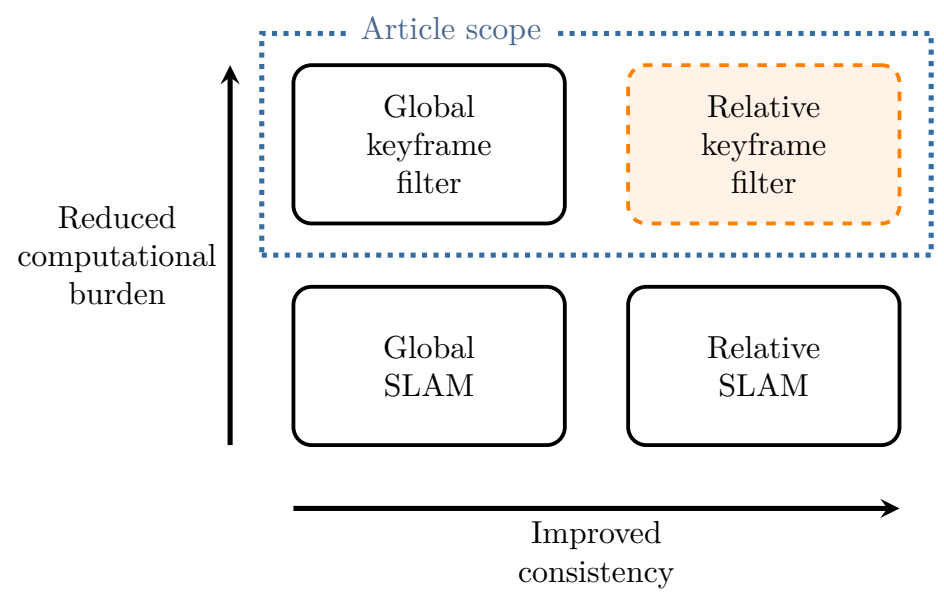

Figure 12: A relative keyframe filter, such as relative navigation, builds upon ideas presented in the SLAM literature to ensure reduced computational complexity and improved consistency. This article compares the relative navigation framework to popular global keyframe filters. 


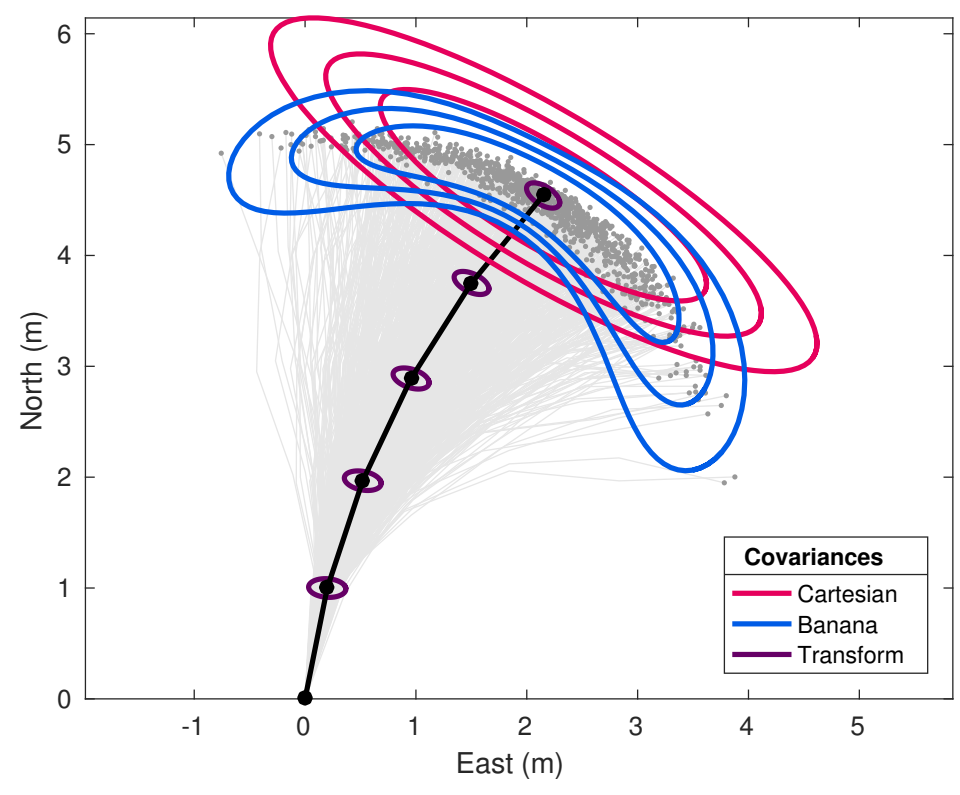

Figure 13: Banana-shaped distribution arising from the concatenation of small, uncertain transforms. The black line shows the nominal path created by concatenating several small transforms with Gaussian uncertainties shown by the purple ellipses. The gray lines and dots show a Monte-Carlo sampling from the transform distributions. The level contours of a Gaussian distribution are shown parameterized with Cartesian coordinates in red, and parameterized with exponential coordinates in blue.

\section{Sidebar: The Banana Distribution}

The "banana distribution," first described in [42], is the sickleshaped distribution of position uncertainty that arises due to heading uncertainty in robot localization problems. Intuition about why the banana distribution occurs can be developed by considering the arc-like distribution that arises for a robot that travels in a straight line for a known distance, but with an uncertain initial heading or growing heading uncertainty.

A similar distribution arises in the pose-graph representation of the relative navigation approach, where several short transforms are concatenated as in the example in Figure 13. Because the length of each individual transform is small and the heading uncertainty is low, the uncertainties on the transforms are well approximated by Gaussian normal distributions. However, when several transforms are concatenated, the resulting global uncertainty becomes distinctly banana shaped. This is illustrated in the distribution shown in Figure 13, created by sampling from the individual transform uncertainties in a Monte-Carlo fashion.

The most common parameterization of uncertainty, inherent to the Kalman filter and its variants, is a Gaussian normal distribution. For filters that estimate the global state directly, the covariance is computed directly in a Cartesian coordinate system. However, this parameterization, illustrated with ellipses in Figure 13, results in a poor fit for the true underlying distribution.

The distribution captured by the pose-graph representation can be better parameterized using a Gaussian normal distribution in exponential coordinates [43]. While methods exist for approximating the final distribution directly from the covariance matrices of the concatenated transforms [23], in this sidebar we discuss computing an approximate distribution from the sample covariance of the Monte-Carlo points, used in [43] and as the baseline method in [23]. To accomplish this, we need to lay some theoretical groundwork.

Rigid body rotations are represented by members of the special orthogonal group,

$$
S O(2) \triangleq\left\{\mathbf{C} \in \mathbb{R}^{2 \times 2} \mid \mathbf{C C}^{\boldsymbol{\top}}=\mathbf{I}, \operatorname{det} \mathbf{C}=1\right\},
$$

for planar motion, or similarly $S O(3)$ for six-degree-offreedom (6DOF) motion. The special Euclidean group,

$$
S E(2) \triangleq\left\{\mathbf{T}=\left[\begin{array}{ll}
\mathbf{C} & \mathbf{r} \\
\mathbf{0} & 1
\end{array}\right] \in \mathbb{R}^{3 \times 3} \mid\{\mathbf{C}, \mathbf{r}\} \in S O(2) \times \mathbb{R}^{2}\right\},
$$

(or $S E(3)$ for $6 \mathrm{DOF}$ ) represents transformations parameterized by a translation $\mathbf{r}$ and rotation $\mathbf{C}$. The pose compounding operations of equations (5) and (6) are equivalent to representing the poses as transforms in $S E(2)$ and multiplying them (or their inverses) as appropriate.

$S E(2)$ is an example of a Lie group, and associated with that group is the Lie algebra $\mathfrak{s e}(2)$. The Lie algebra is the tangent space to the Lie group around the identity element, and unlike the group is a vector space. Members of the Lie algebra can be mapped to elements of the group via the exponential mapping, while the matrix logarithm provides the inverse mapping.

Expressing a difference in pose in exponential coordinates is equivalent to mapping the error transform into the Lie algebra. For each Monte-Carlo point $\mathbf{x}_{i}$ in Figure 13, we therefore express the distance from the true pose $\mathbf{x}$ in exponential coordinates as

$$
\delta \boldsymbol{\xi}_{i}=\log \left(\ominus \mathbf{x} \oplus \mathbf{x}_{i}\right)
$$

where for $S E(2)$

$$
\log (\mathbf{x})=\left[\begin{array}{cc}
\frac{\phi}{2}\left[\begin{array}{cc}
\cot \frac{\phi}{2} & 1 \\
-1 & \cot \frac{\phi}{2}
\end{array}\right]\left[\begin{array}{l}
x \\
y
\end{array}\right] \\
\phi
\end{array}\right]
$$

Level curves of the resulting distribution are shown by the blue lines in Figure 13, and are a much better fit for the distribution represented by the pose graph. This is numerically verified using a log-likelihood ratio test in [43]. Unlike the native pose-graph representation, however, the distribution is parameterized entirely by the mean and covariance, and so additional statistics can easily be computed. This idea is used throughout the article to define the global uncertainty estimate, determine bias, and compute NEES estimates for the relative navigation approach. 


\section{Author Biography}

David O. Wheeler is a Ph.D. student in the Electrical and Computer Engineering Department at Brigham Young University. He received the B.S. degree in mechanical engineering from Brigham Young University in 2013 graduating magna cum laude.

Daniel P. Koch is a Ph.D. student in the Mechanical Engineering Department at Brigham Young University, and a National Defense Science and Engineering (NDSEG) fellow. He received the B.S. degree in mechanical engineering, summa cum laude, with a minor in computer science from Brigham Young University in 2014.

James S. Jackson is a Ph.D. student in the Mechanical Engineering Department at Brigham Young University. He received the B.S. degree in mechanical engineering from Brigham Young University in 2014.

Randal W. Beard received the B.S. degree in electrical engineering from the University of Utah, Salt Lake City, in 1991, the M.S. degree in electrical engineering in 1993, the M.S. degree in mathematics in 1994, and the Ph.D. degree in electrical engineering in 1995, all from Rensselaer Polytechnic Institute, Troy, New York. Since 1996, he has been with the Electrical and Computer Engineering Department at Brigham Young University, Provo, Utah, where he is currently a professor. In 1997 and 1998, he was a Summer faculty fellow at the Jet Propulsion Laboratory, California Institute of Technology, Pasadena. In 2006 and 2007 he was a visiting research fellow at the Air Force Research Laboratory, Munitions Directorate, Eglin Air Force Base, Florida. His primary research focus is autonomous control of miniature air vehicles and multi-vehicle coordination and control. He is a past associate editor for IEEE Control Systems Magazine, the Journal of Intelligent and Robotic Systems, and the IEEE Transactions on Automatic Control. He is a fellow of IEEE.

Timothy W. McLain is a professor in the Department of Mechanical Engineering at Brigham Young University (BYU). He received the B.S. and M.S. degrees in mechanical engineering from BYU. While completing his Ph.D. work at Stanford University, he worked with the Monterey Bay Aquarium Research Institute on the control of underwater robotic vehicles. He joined BYU in 1995. During the summers of 1999 and 2000, he was a visiting scientist at the Air Force Research Laboratory, where he initiated research on unmanned aircraft, an area on which he continues to focus. With Randal Beard, he is the author of the textbook Small Unmanned Aircraft: Theory and Practice (Princeton University Press, 2012). He is currently the director of the Center for Unmanned Aircraft Systems under the National Science Foundation Industry/University Cooperative Research Center program. 